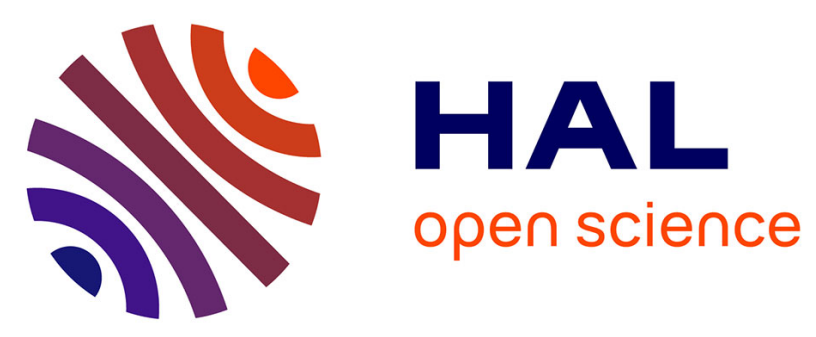

\title{
Formulation variationnelle espace-temps pour le calcul par potentiel retardé de la diffraction d'une onde acoustique (I)
}

\author{
Alain Bamberger, Tuong Ha Duong
}

\section{- To cite this version:}

Alain Bamberger, Tuong Ha Duong. Formulation variationnelle espace-temps pour le calcul par potentiel retardé de la diffraction d'une onde acoustique (I). Mathematical Methods in the Applied Sciences, 1986, 8 (1), pp.405-435. 10.1002/mma.1670080127 . hal-01571259

HAL Id: hal-01571259

https://hal.science/hal-01571259

Submitted on 1 Aug 2017

HAL is a multi-disciplinary open access archive for the deposit and dissemination of scientific research documents, whether they are published or not. The documents may come from teaching and research institutions in France or abroad, or from public or private research centers.
L'archive ouverte pluridisciplinaire HAL, est destinée au dépôt et à la diffusion de documents scientifiques de niveau recherche, publiés ou non, émanant des établissements d'enseignement et de recherche français ou étrangers, des laboratoires publics ou privés. 


\title{
Formulation Variationnelle Espace-Temps pour le Calcul par Potentiel Retardé de la Diffraction d'une Onde Acoustique (I)
}

\author{
A. Bamberger et T. Ha Duong, Palaiseau
}

We give here a space-time variational formula to the problem of the transient acoustic scattering by a free (pressure release) surface, using the retarded potential technique. From this formula, we obtain new schemes for the calculation of the potential density. We prove stability and convergence of such schemes: these are as far as we know, the first results in that direction for such problem. The case of a hard obstacle will be treated in the second part of the paper.

\section{§1. Introduction}

\section{1}

La technique du potentiel retardé est souvent utilisée pour les calculs numériques d'une onde acoustique, élastique ou électromagnétique diffractée par un obstacle illuminé par une onde incidente dans l'espace

R. P. Shaw dans [8] a donné une revue détaillée des travaux avant 1978 dans ce domaine, concernant l'onde acoustique et élastique. Quelques autres travaux publiés après cette date, dont nous avons pris connaissance, par exemple ceux de G. C. Herman [4, 5] avec le potentiel volumique, de C. L. Bennett et H. Mieras [1] ou de l'équipe du C. E. A. J. M. Parot, J. M. Pages, P. Verpeaux [7] avec le potentiel de surface, pas plus que ceux cités par Shaw, ne comportent d'analyse mathématique des schémas de calculs proposés. En particulier, le caractère bien posé du problème traité, les estimations d'erreurs et surtout la stabilité des schémas ne sont pas abordés. Néanmoins, il semble que les résultats numériques obtenus le plus souvent avec des configurations géométriques simples ou possédant des symétries, sont bons. Voir K. N. Mitzner [6], R. P. Shaw et S. A. English [9] ou [1] pour l'onde acoustique et D. M. Cole, D. D. Kosloff, J. B. Minster [3] pour l'onde élastique. Ce dernier travail comporte aussi un début de discussion de la stabilité du schéma dans le cas d'un demiespace. Signalons pour terminer ce survol rapide de bibliographie, l'article de revue de C. L. Bennett et F. J. Ross pour les problèmes électromagnétiques [2]. 
Dans ce travail, nous prenons pour exemple le problème de Dirichlet de l'équation des ondes, en donnons une formulation variationnelle espace-temps qui sert de base pour une nouvelle classe de schémas de calculs stables et convergents.

\subsection{Le problème d'ondes diffractées. Notations}

La figure 1 illustre le problème d'ondes diffractées par un obstacle.
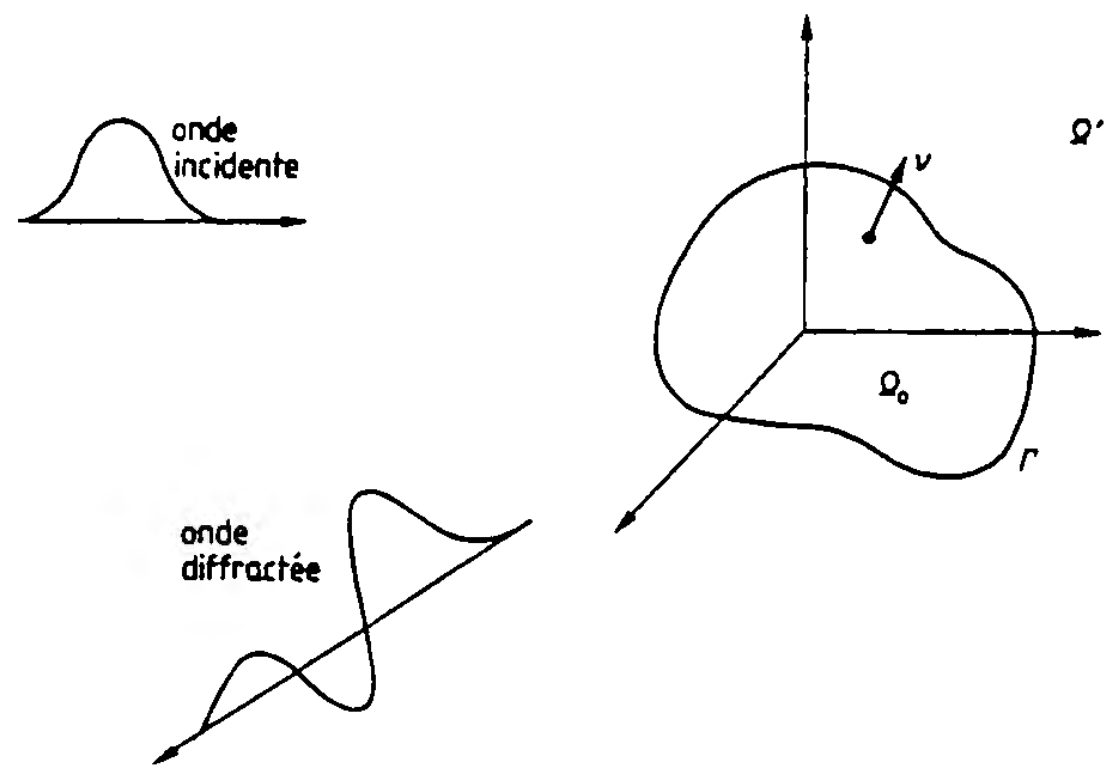

Fig. 1

L'obstacle est un domaine borné $\Omega_{0}$ dans $\mathbf{R}^{3}$, avec une frontière $\Gamma=\partial \Omega$ régulière (voir Ch. $1, \mathrm{n}^{\circ} 7$ de [15]). On note par $v$ le vecteur normal unitaire à $\Gamma$ oriente vers l'extérieur $\Omega^{\prime}=R^{3} \backslash \bar{\Omega}_{0}$ de $\Omega_{0}$. Pour cette raison on notera aussi $\Omega_{-}=\Omega_{0}$ et $\Omega_{+}=\Omega^{\prime}$, et on utilisera la lettre $\Omega$ (sans indice) pour désigner l'un ou l'autre de ces domaines.

L'origine des temps peut être prise en un moment où l'onde incidente n'atteint pas encore l'obstacle, nous avons à déterminer la solution dans $R^{+} \times \Omega^{\prime}$ de l'équation des ondes

$$
\square u=\left(\frac{1}{c^{2}} \frac{\partial^{2}}{\partial t^{2}}-\Delta\right) u(t, x)=f(t, x) \quad\left(t>0, x \in \Omega^{\prime}\right)
$$

ou $f$ est un terme source connu, avec des conditions initiales aussi connues:

$\left.\begin{array}{ll}(1.2) & u(0, x)=u_{0}(x) \\ (1.3) & u_{t}(0, x)=u_{1}(x)\end{array}\right\} \quad\left(x \in \Omega^{\prime}\right)$

et une condition au bord. Pour les problèmes acoustiques, les deux conditions courantes correspondent a une surface libre, ou un obstacle rigide. Ce sont, respectivement les conditions de Dirichlet et de Neumann:

$$
\begin{array}{ll}
u(t, x)=0 & (t>0, x \in \Gamma) \\
\frac{\partial u}{\partial v}(t, x)=0 & (t>0, x \in \Gamma)
\end{array}
$$


Nous nous limitons dans ce travail au problème de Dirichlet (extérieur)

$$
D_{+}=\{(1.1),(1.2),(1.3),(1.4)\}
$$

et pour simplifier l'écriture nous prenons la vitesse $C=1$. Dans un prochain article, nous traiterons le problème de Neumann utilisant un potentiel retardé de double couche.

Sous les hypothèses habituelles pour un problème d'énergie finie

$$
\left\{\begin{array}{l}
f \in C\left(\overline{\mathbf{R}}^{+}, L^{2}\left(\Omega^{\prime}\right)\right) ; \quad f(0, \cdot)=0 \\
u_{0} \in H_{0}^{1}\left(\Omega^{\prime}\right) \text { et } u_{1} \in L^{2}\left(\Omega^{\prime}\right)
\end{array}\right.
$$

la solution $u$ de $\left(D_{+}\right)$peut être mise sous forme de la somme d'une onde incidente $u^{\text {inc }}$ et d'une onde diffractée $u^{\text {sc }}$. La première sera la restriction à $R^{+} \times \Omega^{\prime}$ de l'onde $\dot{u}$ définie dans l'espace libre $\mathbf{R} \times \mathbf{R}^{3}$ par

$$
\left\{\begin{array}{l}
\square \dot{u}=\bar{f} \\
\dot{u}(0, \cdot)=\bar{u}_{0}(\cdot) \\
\dot{u}_{t}(0, \cdot)=\bar{u}_{1}(\cdot)
\end{array}\right.
$$

où $\overline{f,} \bar{u}_{0}$ et $\bar{u}_{1}$ sont respectivement les prolongements par 0 de $f, u_{0}, u_{1}$ dans les complémentaires de leurs domaines de définition. Ce qui donne $f \in C\left(\boldsymbol{R}, L^{2}\left(\boldsymbol{R}^{3}\right)\right)$, $\bar{u}_{0} \in H^{1}\left(\mathbf{R}^{3}\right)$ et $\bar{u}_{1} \in L^{2}\left(\mathbf{R}^{3}\right)$.

Rappelons alors (cf. Trèves [18]) que $\dot{u}$ peut être explicitée en fonction de $\bar{f}, \bar{u}_{0}, \bar{u}_{1}$ et a la régularité exprimée par:

$$
\dot{u} \in C\left(\mathrm{R}, H^{1}\left(\mathrm{R}^{3}\right)\right) \cap C^{1}\left(\mathrm{R}, H^{0}\left(\mathbf{R}^{3}\right)\right) .
$$

L'onde diffractée $u^{2 c}=u-\left.\dot{u}\right|_{\mathrm{R}+\times \Omega^{\prime}}$ est alors solution du problème mixte non homogene suivant

$$
\left\{\begin{array}{l}
\square u^{\text {sc }}=0 \quad \text { dans } \mathbf{R}^{+} \times \Omega^{\prime} \\
u^{\text {sc }}(0)=0 \\
u_{t}^{\text {sc }}(0)=0 \\
u^{\text {sc }}(t, x)=g(t, x) \text { sur } \mathbf{R}^{+} \times \Gamma
\end{array}\right.
$$

où $g=-\left.\dot{u}\right|_{\mathrm{R}^{+} \times \Gamma} \in C\left(\overline{\mathbf{R}}^{+}, H^{1 / 2}(\Gamma)\right)$ et $g(0)=0$.

D'autres conditions sur $f, u_{0}, u_{1}$ donnant plus de régularité pour $\dot{u}$ donc pour $g$ se trouvent aussi dans [18].

\subsection{Potentials retardés de surface}

C'est le problème (I) que nous allons résoudre dans la suite. On peut bien sûr, trouver plusieurs résultats concernant ce problème dans les monographies traitant les équations aux dérivées partielles (cf. par exemple Lions-Magenes [15, Ch. 4], et Charazain-Piriou [13, Ch. 7]). Notre but est plus numérique, et passe par une formule de représentation de la solution. 
Rappelons pour cela la formule de Kirchoff:

$$
\begin{aligned}
\varepsilon u(t, x)= & \frac{1}{4 \pi} \int_{\Gamma}\left\{-\frac{1}{|x-y|} \frac{\partial u}{\partial v}(\tau, y)+\frac{(x-y) \cdot v_{y}}{|x-y|^{2}}\right. \\
& \left.\cdot\left(\frac{u(\tau, y)}{|x, y|}+\frac{\partial u}{\partial t}(\tau, y)\right)\right\} \mathrm{d} \sigma_{y}
\end{aligned}
$$

où; pour $t>0, \tau=t-|x-y|$ et

$$
\varepsilon=\left\{\begin{array}{cc}
1 & \text { si } x \in \Omega^{\prime} \\
0 & x \in \Omega_{0} . \\
\frac{1}{2} & x \in \Gamma
\end{array}\right.
$$

Formule valable pour $u$ vérifiant $\square u=0$ dans $\mathrm{R} \times \Omega^{\prime}$ et $u(t, \cdot) \equiv 0 \mathrm{si}$ $t \leqslant 0$. On prolonge alors $u^{\text {sc }}$ par 0 pour $t \leqslant 0$ pour l'appliquer.

On peut utiliser (1.6) pour représenter la solution de (I), après avoir résolu l'équation intégrale de variable $\partial u^{s c} / \partial v$

$$
\left\{\begin{aligned}
& \frac{1}{2} g(t, x)= \frac{1}{4 \pi} \int_{\Gamma} \frac{1}{|x-y|} \frac{\partial u^{s c}}{\partial \nu}(\tau, y) \mathrm{d} \sigma_{y} \\
&+\frac{1}{4 \pi} \int_{\Gamma} \frac{(x-y) \cdot v_{y}}{|x-y|^{2}}\left[\frac{g(\tau, y)}{|x-y|}+\frac{\partial g}{\partial t}(\tau, y)\right] \mathrm{d} \sigma_{y} \\
& \text { pour } t>0, x \in \Gamma .
\end{aligned}\right.
$$

Nous préférons, à la place de la formule de Kirchoff, chercher comme pour les équations de Laplace ou de Helmholtz - une représentation de $u^{\text {sc }}$ par un potentiel retardé de simple couche:

$$
u^{s c}(t, x)=\frac{1}{4 \pi} \int_{\Gamma} \frac{\varphi(t-|x-y|, y)}{|x-y|} \mathrm{d} \sigma_{y} \quad(t>0, x \notin \Gamma)
$$

ce qui revient, c'est bien connu ([10], [12] resp. pour les équations de Laplace et de Helmholtz), à considérer en même temps que le problème (I) un problème intérieur avec la même donnée frontière $g$, puis à prendre pour $\varphi$ le saut de $\partial u / \partial v$ traversant $\Gamma$ de $\Omega_{0}$ à $\Omega^{\prime}$.

Cette densité inconnue $\varphi$ sera alors solution de l'équation intégrale

$$
g(t, x)=\frac{1}{4 \pi} \int_{\Gamma} \frac{\varphi(\tau, y)}{|x-y|} \mathrm{d} \sigma_{y} \quad(t>0, x \in \Gamma) .
$$

Nous traiterons ce problème en passant par une transformation de FourierLaplace en temps: cela donne un problème relatif à l'équation de Helmholtz $\left(\Delta+\omega^{2}\right) u=0$ avec une fréquence $\omega$ complexe dont l'étude est faite au $\S 2$. Au $\$ 3$, nous inversons la transformation, obtenons une formulation variationnelle en temps et en espace de l'équation (1.7), avec une propriété de coercivité de la 
forme bilinéaire concernée. Les schémas numériques pour résoudre le problème variationnel ainsi obtenu sont présentés au $\S 4$, leur analyse aux $\S 5$ et 6 . Voir les résultats aux numéros $5.3,5.4$ et au $\S 6$.

\section{$\$ 2$ Équation de Helmholtz avec $\omega$ complexe}

\subsection{Les problèmes transformés}

Récrivons ici le problème (I) (en supprimant désormais l'indice sc):

$$
\left(P_{+}\right)\left\{\begin{array}{l}
\square u=0 \\
u(0, \cdot)=0 \\
u_{t}(0, \cdot)=0 \\
\left.u\right|_{R^{+} \times \Gamma}=g
\end{array} \quad \text { dans } R^{+} \times \Omega_{+}\right.
$$

ainsi que le problème intérieur associé:

$$
\left(P_{-}\right)\left\{\begin{array}{l}
\square u=0 \quad \text { dans } \mathbf{R}^{+} \times \Omega_{-} \\
u(0, \cdot)=0 \\
u_{t}(0, \cdot)=0 \\
\left.u\right|_{\mathbf{R}^{+} \times \Gamma}=g .
\end{array} \text { dans } \Omega_{-}\right.
$$

Supposons que $g$ et $u$ prolongées par 0 pour $t<0$ soient des distributions de "Laplace transformables" sur $\mathrm{R}$, a valeurs respectivement dans $H^{1 / 2}(I)$ et $H^{1}\left(\Omega_{+}\right) \cup H^{1}\left(\Omega_{-}\right)$(c'est-à-dire qu'il existe un $\sigma_{0} \in \mathbf{R}$ tel que $\mathrm{e}^{-\sigma_{0} t} g$ et $\mathrm{e}^{-\sigma_{0} t} u$ soient des distributions tempérées sur $\mathbf{R}$, à valeurs dans ces espaces). Nous posons

$$
\hat{g}(\omega, \cdot)=\int_{R} \mathrm{e}^{\mathrm{i} \omega t} g(t, \cdot) \mathrm{d} t
$$

pour $\operatorname{Im} \omega>\sigma_{0}$. De même pour $\hat{u}(\omega, \cdot)$, qui sera solution des problèmes transformés suivants pour tout $\omega$ de ce dernier plan $\left\{\operatorname{Im} \omega>\sigma_{0}\right\}$ :

$$
\left(P_{ \pm}^{\omega}\right) \begin{cases}\mathfrak{a}(\omega, \cdot) \in H^{1}\left(\Omega_{ \pm}\right) & \\ \left(\Delta+\omega^{2}\right) \hat{u}(\omega, \cdot)=0 & \text { dans } \Omega_{ \pm} \\ \hat{u}(\omega, \cdot)=\hat{g}(\omega, \cdot) & \text { sur } \Gamma .\end{cases}
$$

Il est bien connu que sur le demi-plan $\{\operatorname{Im} \omega>0\}$ (on peut toujours prendre $\sigma_{0} \geqslant 0$ !) les problèmes $\left(P_{ \pm}^{\omega}\right)$ admettent chacun une solution unique - pour le problème extérieur $\left(P_{+}^{\omega}\right)$, la condition $\hat{u}(\omega, \cdot) \in H^{1}\left(\Omega_{+}\right)$remplace la condition de radiation a l'infini de Sommerfeld. Mais, en vue de faire la transformation de Laplace inverse, nous reprenons dans ce paragraphe la résolution de ces problèmes, pour préciser les dépendances en $\omega$ et $a$ et de ses traces normales $(\partial u / \partial v)_{ \pm}$.

Les résultats essentiels pour la suite sont dans les propositions 1 et 3 cidessous. 
Nous noterons $\|\cdot\|_{s, \Omega_{ \pm}}$la norme de $H^{s}\left(\Omega_{ \pm}\right)$et $|\cdot|_{s}$ celle de $H^{s}(\Gamma)$, et nous supprimerons pour alléger l'écriture le chapeau $(\hat{\gamma})$ sur $\hat{u}(\omega, \cdot) \ldots$ chaque fois qu'il n'y a pas de risque de confusion - en particulier si $\omega y$ figure en toute lettre.

\subsection{Un lemme de relèvement}

Lemme 1. Pour tout $\psi \in H^{1 / 2}(I)$ et $\omega \in\left\{\operatorname{Im} \omega>\sigma_{0}>0\right\}$, il existe $v(\omega) \in H^{1}(\Omega)$ tel que $\left.v\right|_{\Gamma}=\omega$ et

(2.1) $\int_{\Omega}\left(|\nabla v|^{2}+|\omega v|^{2}\right) \mathrm{d} x \leqslant C \max \left(\frac{1}{\sigma_{0}}, 1\right)|\omega||\psi|_{\mathbf{i} / 2}^{2}$

où $C$ ne dépend que de la géométrie de $\Gamma$.

Démonstration. En passant par une partition de l'unité si nécessaire, on peut supposer que le support de $\psi$ soit inclus dans $\Gamma \cap U$, où $U$ est le domaine d'une carte $\chi$ au bord de $\Omega$, i.e. un difféomorphisme de $U \cap \Omega$ dans $\tilde{U} \cap \mathbf{R}_{+}^{3}$ où $\tilde{U}$ est l'ouvert $\left\{\left(y^{\prime}, y_{3}\right) ;\left|y^{\prime}\right|<1,\left|y_{3}\right|<1\right\}$ de $\mathbf{R}^{3}$, et de plus $\left.\chi\right|_{U \cap \Gamma}$ est un difféomorphisme de $U \cap \Gamma$ dans $U \cap\left\{y_{3}=0\right\}$.

La norme $H^{1 / 2}(\Gamma)$ de $\psi$ est équivalente à la norme $H^{1 / 2}\left(\mathrm{R}^{2}\right)$ de $\tilde{\psi}=\psi_{0}\left(\left.\chi\right|_{U \cap \Gamma}\right)^{-1}$. D'autre part, si $v \in H^{1}(\Omega)$, à support dans $U \cap \Omega$, et $\tilde{v}=v_{0} \chi^{-1}$, on a:

$$
\begin{aligned}
\int_{\Omega}\left(|\nabla v|^{2}+|\omega v|^{2}\right) \mathrm{d} x= & \left.\left.\int_{\tilde{U} \cap \mathrm{R}_{+}^{3}}\left(\mid J(x)^{-1}(y)\right) \cdot \nabla \tilde{v}(y)\right|^{2}+|\omega \tilde{v}(y)|^{2}\right) \\
& \cdot\left|J\left(\chi^{-1}\right)(y)\right| \mathrm{d} y
\end{aligned}
$$

où $J\left(\chi^{-1}\right)$ est le jacobien, qui est borné sur $\tilde{U} \cap \mathbf{R}_{+}^{3}$. D'où:

$$
\int_{\Omega}\left(|\nabla v|^{2}+|\omega v|^{2}\right) \mathrm{d} x \leqslant C_{1} \int_{\left[U \cap \mathrm{R}_{+}^{3}\right.}\left(|\nabla \tilde{v}(y)|^{2}+|\omega \tilde{v}(y)|^{2}\right) \mathrm{d} y .
$$

Soit $W\left(y^{\prime}, y_{3} ; \omega\right)$ la solution dans $H^{1}\left(\mathbf{R}_{+}^{3}\right)$ du problème

$$
\begin{aligned}
\left(-\Delta+|\omega|^{2}\right) W & =0 \\
\left.W\right|_{y_{3}=0} & =\tilde{\psi}
\end{aligned}
$$

solution dont la transformée de Fourier partielle en $y^{\prime}$ est donnée par:

$$
\hat{W}\left(\xi^{\prime}, y_{3} ; \omega\right)=\hat{\tilde{\psi}}\left(\xi^{\prime}\right) e^{-y_{3} \sqrt{\left|\xi^{\prime}\right|^{2}+|\omega|^{2}}} \quad y_{3}>0
$$

on a immédiatement

$$
\begin{aligned}
\int_{\mathbf{R}_{+}^{3}}\left(|\nabla w|^{2}+|\omega w|^{2}\right) \mathrm{d} y & =(2 \pi)^{2} \int_{\mathbf{R}^{2}} \mathrm{~d} \xi^{\prime} \int_{0}^{\infty}\left\{\left(\left|\xi^{\prime}\right|^{2}+|\omega|^{2}\right)|\hat{w}|^{2}+\left|\frac{\partial \hat{w}}{\partial y_{3}}\right|^{2}\right\} \mathrm{d} y_{3} \\
& =(2 \pi)^{2} \int_{\mathbf{R}^{2}}\left(\left|\xi^{\prime}\right|^{2}+|\omega|^{2}\right)^{1 / 2}\left|\hat{\tilde{\psi}}\left(\xi^{\prime}\right)\right|^{2} \mathrm{~d} \xi^{\prime} .
\end{aligned}
$$


Posant $\omega=\eta+\mathrm{i} \sigma$, et remarquant que

(2.2) $\left|\xi^{\prime}\right|^{2}+\eta^{2}+\sigma^{2} \leqslant \max \left(\frac{1}{\sigma_{0}^{2}}, 1\right)\left(\eta^{2}+\sigma^{2}\right)\left(1+\left|\xi^{\prime}\right|^{2}\right) \quad \forall \sigma \geqslant \sigma_{0}$ on en déduit que pour tout $\omega \in\left\{\operatorname{Im} \omega \geqslant \sigma_{0}>0\right\}$ :

$$
\int_{R_{+}^{3}}\left(|\nabla w|^{2}+|\omega w|^{2}\right) \mathrm{d} x \leqslant(2 \pi)^{2} \max \left(\frac{1}{\sigma_{0}}, 1\right)|\omega||\bar{\psi}|_{1 / 2}^{2} .
$$

Il suffit maintenant de prendre $v=\bar{v}_{0} \chi$ avec

$$
\bar{v}=w(y ; \omega) \theta\left(y_{3}\right),
$$

$\theta \in \boldsymbol{C}_{0}^{\infty}\left(\overline{\mathbf{R}}_{+}\right), \theta(0)=1$ et $\operatorname{supp} \theta \subset[0,1[$.

Remarque. Considérant le problème

$$
\left\{\begin{aligned}
\left(-\Delta+|\omega|^{2}\right) v_{1} & =0 \quad \text { dans } \Omega \\
\left.v_{1}\right|_{r} & =\psi \in H^{1 / 2}(\Gamma)
\end{aligned}\right.
$$

on peut voir facilement que la norme

$$
\left\|v_{1}\right\|_{\Omega, \omega}=\left(\int_{\Omega}\left|\nabla v_{1}\right|^{2}+\left|\omega v_{1}\right|^{2}\right)^{1 / 2}
$$

est minimale parmi les relèvements dans $H^{1}(\Omega)$ de $\psi$.

D'autre part, l'inégalité (2.2) est aussi "la meilleure possible» (faire par exemple $\eta=0$ et $\sigma \rightarrow \infty$ ), l'inégalité (2.1) est donc optimale au sens où aucune puissance de $|\omega|$ plus petite que 1 ne peut être obtenue au $2^{\mathrm{d}}$ membre de (2.1).

Comme conséquence du lemme 1 , nous obtenons la majoration cidessous pour les solutions des problèmes $\left(P_{ \pm}^{\omega}\right)$.

Proposition 1. Le problème $P_{+}^{\omega}$ (resp. $P_{-}^{\omega}$ ) admet une solution unique dans $H^{1}\left(\Omega_{+}\right)\left(\right.$resp. $\left.H^{1}\left(\Omega_{-}\right)\right)$pour tout $\omega$ avec $\operatorname{Im} \omega>0$. Cette solution vérifie:

$$
\|u\|_{\substack{\Omega_{+}, \omega \\(-)}}^{2} \leqslant C \frac{1}{\sigma^{2}} \max \left(\frac{1}{\sigma_{0}}, 1\right)|\omega|^{3}|g|_{1 / 2}^{2}
$$

pour tout $\omega \in\left\{\operatorname{Im} \omega=\sigma \geqslant \sigma_{0}>0\right\}$. C étant une constante ne dépendant que de $\Gamma$.

Démonstration. On résoud $\left(P_{+}^{\omega}\right)$ avec la méthode variationnelle:

Si $v_{0}$ est un relèvement dans $H^{1}\left(\Omega_{+}\right)$de $g, u$ est solution de $\left(P_{+}^{\omega}\right)$ si et seulement si

$$
a\left(u-v_{0}, v\right)=-a\left(v_{0}, v\right) \quad \forall v \in H_{0}^{1}\left(\Omega_{+}\right)
$$

où la forme a définie sur $H^{1}\left(\Omega_{+}\right) \times H^{1}\left(\Omega_{+}\right)$par

$$
a\left(v_{1}, v_{2}\right)=\int_{\Omega_{+}}\left(-\nabla v_{1} \cdot \nabla \bar{v}_{2}+\omega^{2} v_{1} \bar{v}_{2}\right) \mathrm{d} x
$$


vérifie la relation de coercivité:

$$
\sigma \int_{\Omega_{+}}\left(|\nabla v|^{2}+|\omega v|^{2}\right) \mathrm{d} x=\operatorname{Im} \bar{\omega} a(v, v) \leqslant|\omega||a(v, v)| .
$$

La quantité

$$
\|v\|_{\Omega_{+}, \omega}=\left(\int_{\Omega_{+}}\left(|\nabla v|^{2}+|\omega v|^{2}\right) \mathrm{d} x\right)^{1 / 2}
$$

est évidemment une norme équivalente dans $H_{1}\left(\Omega_{+}\right)$si $\omega \neq 0$.

Appliquant l'inégalité de Cauchy-Schwarz au $2^{\mathrm{d}}$ membre de

$$
a(u, u)=a\left(u, v_{0}\right)
$$

et tenant compte de (2.4), on obtient

$$
\|u\|_{\Omega_{+}, \omega} \leqslant \frac{|\omega|}{\sigma}\left\|v_{0}\right\|_{\Omega_{+}, \infty} .
$$

D'où (2.3) si $v_{0}$ est choisi selon le lemme 1.

Pour $\left(P_{-}^{\omega}\right)$, c'est la même chose.

\subsection{Représentation de $\boldsymbol{u}$ par un potentiel de simple couche}

Selon les formules de représentation classiques pour les solutions de l'équation de Helmholtz (dont la validité dans le cas $\operatorname{Im} \omega>0, u \in H^{1}\left(\Omega_{+}\right)$, est simple à vérifier), on peut maintenant écrire, si $u$ résoud à la fois $\left(P_{+}^{\omega}\right)$ et $\left(P_{-}^{\omega}\right)$ et $u \in C^{1}\left(\bar{\Omega}_{+}\right) \cap C^{1}\left(\bar{\Omega}_{-}\right)$:

$$
u(\omega, x)=\frac{1}{4 \pi} \int_{\Gamma} \frac{e^{\mathrm{i} \omega|x-y|}}{|x-y|} \varphi(\omega, y) \mathrm{d} \sigma_{y} \quad\left(x \in \Omega_{+} \cup \Omega_{-}\right)
$$

avec

$$
\varphi(\omega, \cdot)=\left\lceil\frac{\partial u(\omega, \cdot)}{\partial v}\right\rceil
$$

où le crochet [ ] désigne le saut $(\partial u / \partial v)_{-}-(\partial u / \partial v)_{+}$.

Par continuité à travers $\Gamma$ du potentiel (2.5), on obtient l'équation intégrale pour $\varphi$ :

$$
g(\omega, x)=\frac{1}{4 \pi} \int_{\Gamma} \frac{\mathrm{e}^{\mathrm{i} \omega|x-y|}}{|x-y|} \varphi(\omega, y) \mathrm{d} \sigma_{y} \quad(x \in \Gamma) .
$$

Désignons par $S_{\omega}$ l'opérateur intégral au second membre de (2.6) et par $N^{\omega}$ l'opérateur associant à $g$ le saut $[\partial u / \partial \nu]$ de la solution de $\left(P_{+}^{\omega}\right) \cup\left(P_{-}^{\omega}\right)$.

Il est connu ([10]) que $S_{\omega}$ est, quelque soit $\omega$, un opérateur pseudo-différentiel d'ordre -1 sur $\Gamma$. Pour $\operatorname{Im} \omega>0$, nous obtenons un peu plus de précisions sur $S_{w}$, en particulier une relation de coercivité permettant de résoudre (2.6) numériquement avec la méthode variationnelle. Commençons par: 
Proposition 2. Pour $\operatorname{Im} \omega>0$, l'opérateur $N^{\omega}$ est un isomorphisme de $H^{1 / 2}(\Gamma)$ dans $H^{-1 / 2}(\Gamma)$, de norme vérifiant la majoration:

(2.7) $\left\|N^{\omega}\right\| \leqslant C \frac{1}{\sigma_{0}} \max \left(\frac{1}{\sigma_{0}}, 1\right)|\omega|^{2} \quad \forall \omega \in\left\{\operatorname{Im} \omega \geqslant \sigma_{0}>0\right\}$.

Il vérifie la relation de coercivité suivante:

(2.8) $\operatorname{Re}\left\langle N^{\omega} g,-\mathrm{i} \omega g\right\rangle \geqslant C \sigma_{0} \min \left(\sigma_{0}^{2}, 1\right)|g|_{1 / 2}^{2}\left\{\begin{array}{l}\forall g \in H^{1 / 2}(\Gamma) e t \\ \forall \omega \in\left\{\operatorname{Im} \omega \geqslant \sigma_{0}>0 !\right.\end{array}\right.$

où le crochet est l'antidualité entre $H^{-1 / 2}(\Gamma)$ et $H^{1 / 2}(\Gamma)$. Les constantes dans (2.7) et (2.8) ne dépendent que de $\Gamma$.

Démonstration. Les formules de Green:

$$
\begin{aligned}
& \int_{\Omega_{+}} \frac{\partial u_{+}}{\partial v} \cdot \bar{v} \mathrm{~d} \sigma=-\int_{\Omega_{+}}\left(\nabla u \cdot \nabla \bar{v}-\omega^{2} u \cdot \bar{v}\right) \mathrm{d} x \\
& \int_{\Omega_{-}} \frac{\partial u_{-}}{\partial v} \cdot \bar{v} \mathrm{~d} \sigma=+\int_{\Omega_{-}}\left(\nabla u \cdot \nabla \bar{v}-\omega^{2} u \cdot \bar{v}\right) \mathrm{d} x
\end{aligned}
$$

valables pour $u$ solution de $\left(P_{ \pm}^{\omega}\right)$ et $v \in H^{1}\left(\Omega_{+}\right)$(resp. $H^{1}\left(\Omega_{-}\right)$) permettent en effet de définir $\partial u_{+} / \partial v$ et $\partial u_{-} / \partial v$ dans $H^{-1 / 2}(\Gamma)$. L'inégalité de CauchySchwarz donne ensuite:

$$
\left|\int_{\Gamma} \frac{\partial u_{+}}{\partial v} \cdot \bar{\psi} \mathrm{d} \sigma\right| \leqslant\|u\|_{\Omega_{+}, \omega}\|v\|_{\Omega_{+}, \omega}
$$

pour tout $\psi \in H^{1 / 2}(\Gamma)$ et $v \in H^{1}\left(\Omega_{+}\right)$relevant $\psi$. Le lemme 1 permet alors d'obtenir l'inégalité:

$$
\left|\frac{\partial u_{+}}{\partial v}\right|_{-1 / 2} \leqslant C \max \left(\frac{1}{\sigma_{0}^{1 / 2}}, 1\right)|\omega|^{1 / 2}\|u\|_{\Omega_{+}, \omega}
$$

pour tout $\omega \in\left\{\operatorname{lm} \omega \geqslant \sigma_{0}>0\right\}$. De même pour $\partial u_{-} / \partial v$. Jointes à (2.3), ces estimations donnent bien (2.7).

Avec $v=-\mathrm{i} \omega u$ dans les formules de Green, on a d'autre part:

$$
\operatorname{Re} \int_{\Gamma} \frac{\partial u}{\partial v} \cdot(+\mathrm{i} \bar{\omega} \bar{g}) \mathrm{d} \sigma=\sigma \Omega_{\Omega_{+}}\left(\left.\left|\nabla u \Omega^{2}+\right| \omega u\right|^{2}\right) \mathrm{d} x .
$$

On conclut à (2.8) par le théorème de trace. L'inversibilité de $N^{\omega}$ en résulte.

Proposition 3. Pour $\operatorname{Im} \omega>0$, $S_{\omega}$ est l'inverse de $N^{\omega}$ de $H^{-1 / 2}(\Gamma)$ à $H^{1 / 2}(\Gamma)$. Il vérifie la relation de coercivité suivante:

(2.10) $\operatorname{Re}\left\langle\varphi,-\mathrm{i} \omega S_{\omega} \varphi\right\rangle \geqslant C \min \left(\sigma_{0}, 1\right) \frac{|\varphi|_{-1 / 2}^{2}}{|\omega|} \quad \forall \varphi \in H^{-1 / 2}(\Gamma)$

ainsi que la majoration: 
(2.11) $\left\|S_{\omega}\right\| \leqslant C|\omega| \frac{1}{\sigma_{0}} \max \left(\frac{1}{\sigma_{0}^{2}}, 1\right)$

pour tout $\omega \in\left\{\operatorname{Im} \omega \geqslant \sigma_{0}>0\right\}$.

Démonstration. Les résultats de régularité elliptique permettent en effet de valider les relations (2.5) et (2.6) pour $g$ régulière. Par densité, on obtient ensuite l'identité $S_{\omega} N^{\omega}=$ Id sur $H^{1 / 2}(\Gamma)$. La proposition 2 donne alors $N^{\omega} S_{\omega}$ $=$ Id sur $H^{-1 / 2}(\Gamma)$.

Partant de $\varphi \in H^{-1 / 2}(I)$, on peut donc construire $u$ solution de $\left(P_{ \pm}^{\omega}\right)$ avec donnée $g=S_{\omega} \varphi$. La formule de Green peut alors s'écrire sous la forme

(2.12) $\operatorname{Re}\left\langle\varphi, \mathrm{i} \omega S_{\omega} \varphi\right\rangle=\sigma\left(\|u\|_{\Omega_{+}, \omega}^{2}+\|u\|_{\Omega_{-}, \omega}^{2}\right)$.

D'où (2.10) par suite de (2.9).

Quant à (2.11), cela résulte de (2.8).

Ainsi, la solution $u$ de $\left(P_{ \pm}^{\omega}\right)$ admet une et une seule représentation par un potentiel de simple couche (2.5). Sa densité $\varphi$ est solution du problème variationnel

$$
\left\{\begin{array}{l}
\varphi \in H^{-1 / 2}(\Gamma) \\
\left\langle\psi,-i \omega S_{\omega} \varphi\right\rangle=\langle\psi,-i \omega g\rangle \quad \forall \psi \in H^{-1 / 2}(\Gamma)
\end{array}\right.
$$

équivalente à l'équation (2.6).

Signalons enfin l'inégalité évidente suivante qui sera utilisée dans la suite

$$
\left|S_{\omega} \varphi\right|_{L^{2}(\Pi)} \leqslant|\varphi|_{L^{2}()} \quad \forall \varphi \in L^{2}(\Gamma), \operatorname{Im} \omega \geqslant 0
$$

où $C$ ne dépend que de $\Gamma$.

\section{§3 Formulation variationnelle espace-temps pour le potentiel retardé}

Nous revenons dans ce paragraphe au problème $P_{+}\left(\mathrm{cf} . \mathrm{n}^{\circ} 2.1\right)$ et à la recherche d'une représentation de sa solution par un potentiel retardé de simple couche. Nous démontrons, dans un cadre fonctionnel de type Sobolev, l'existence et l'unicité de ce potentiel (th. 2) et donnons ensuite une formulation variationnelle pour calculer sa densité (cf. (3.6) ci-dessous).

Afin d'appliquer la transformation de Laplace inverse aux résultats du $\S 2$, commençons par queleques rapples sur cette transformation. Cf. Trèves [18].

\subsection{Rapples et notations}

Si $E$ est un espace du Banach, on note par $D_{+}^{\prime}(E)$ l'espace des distributions sur $R$, à valeurs dans $E$ et nulles pour $t<0$, puis par $S_{+}^{\prime}(E)$ les distributions tempérées dans $D_{+}^{\prime}(E)$. On pose ensuite

$$
L^{\prime}(E)=\left\{f \in D_{+}^{\prime}(E) \text { telle que } \mathrm{e}^{-\sigma_{0} t} f \in S_{+}^{\prime}(E) \text { pour un } \sigma_{0}(f) \in R_{\}} .\right.
$$


Il est clair que si $\mathrm{e}^{-\sigma_{0} t} f \in S_{+}^{\prime}(E)$, alors $\mathrm{e}^{-\sigma t} f \in S_{+}^{\prime}(E)$ pour tout $\sigma>\sigma_{0}$, et on peut poser pour $\omega=\eta+\mathrm{i} \sigma, \sigma \geqslant \sigma_{0}$ :

$$
f(\omega)=L f(\omega)=\int_{-\infty}^{+\infty} e^{\mathrm{j} \omega t} f(t) \mathrm{d} t=F\left(\mathrm{e}^{-\sigma t} f\right)(\eta) .
$$

- On a modifié ici la variable de Laplace pour respecter les conventions d'écritures habituelles des ondes.

L'équivalence suivante est fondamentale:

$(L)$

une fonction $\hat{f}(\omega)$ est la transformée de Laplace d'une distribution $f \in L^{\prime}(E)$ - i.e. vérifie (3.1) - si et seulement si elle est définie, holomorphe dans un demi-plan complex $\left\{\operatorname{Im} \omega>\sigma_{0}^{\prime}\right.$; à valeurs dans $E$, et majorée par un polynôme en $|\omega|$ dans un demi-plan fermé plus petit. Ceci signifie l'existence des constantes $\sigma_{1}>\sigma_{0}^{\prime}, C>0$ et un entier $k \geqslant 0$ tels que

$\|\hat{f}(\omega)\|_{E} \leqslant C(1+|\omega|)^{k} \quad \forall \omega \in\left\{\operatorname{Im} \omega \geqslant \sigma_{1}^{\prime},\right.$.

Elle nous permet déjà d'énoncer un théorème général sur la solution de $\left(P_{+}\right)$et sa représentation par un potentiel retardé.

Théorème 1. Pour tout $g$ appartement à $L^{\prime}\left(H^{1 / 2}(\Gamma)\right)$, le problème $\left(P_{+}\right)$admet une solution unique dans $L^{\prime}\left(H^{1}\left(\Omega_{+}\right)\right)$, solution représentable d'une et d'une seule façon comme un potentiel retardé de simple couche sur $\Gamma$. La densité de ip de ce potentiel appartient à $L^{\prime}\left(H^{-1 / 2}(\Gamma)\right)$.

Démonstration. Utilisant le noyau de $S_{\omega}$, on vérifie sans peine que la fonction $\left(\omega \rightarrow S_{\omega}\right)$ définie sur $\mathrm{C}$, à valeurs dans $L\left(H^{-1 / 2}(\Gamma), H^{1 / 2}(\Gamma)\right)$ est holomorphe. Il en est de même donc de $N^{\omega}=\left(S_{\omega}\right)^{-1}$ sur le demi-plan $\{\operatorname{Im} \omega>0\}$. Par conséquent, si $\hat{g}$ est holomorphe sur un demi-plan $\left\{\operatorname{Im} \omega>\sigma_{0}\right.$ ', à valeurs dans $H^{1 / 2}(\Gamma)$, la solution $\phi(\omega)$ de l'équation $S_{\omega} \hat{\varphi}=\hat{g}$ qui existe pour $\operatorname{Im} \omega>0$ est une fonction holomorphe sur le demi-plan $\left\{\operatorname{Im} \omega>\left(\sigma_{0} \vee 0\right)\right.$ à valeurs dans $H^{-1 / 2}(\Gamma)$. La représentation (2.5) permet alors de voir que $\hat{u}(\omega, \cdot)$ est aussi holomorphe sur le même demi-plan et à valeurs dans $H^{1}\left(\Omega_{+}\right)$.

Les majorations (2.3) et (2.7) permettent de voir que $\hat{a}$ et $\hat{\phi}$ vérifient bien $(L)$, donc sont les transformations de Laplace des distributions dans $L\left(H^{1}\left(\Omega_{+}\right)\right)$ et $L^{\prime}\left(H^{-1 / 2}(\Gamma)\right)$, qui sont respectivement solution de $\left(P_{+}\right)$et sa densité du potentiel retardé.

Mais, en vue d'exploiter les inégalités de coercivité obtenues au $\$ 2$, nous allons mettre plus d'accent sur des résultats dans un cadre hilbertien, utilisant en particulier la formule de Parseval. Commençons par définir des espaces de type Sobolev:

$$
H_{\sigma}^{s}\left(\mathbf{R}_{+}, E\right)=\left\{f \in L^{\prime}(E) ; \mathrm{e}^{-\sigma t} \Lambda^{s} f \in L^{2}\left(\mathbf{R}_{t}, E\right)\right\}
$$

où $\sigma>0, s \in \mathbf{R}$ et l'opérateur $\Lambda^{s}$ défini de $L^{\prime}(E)$ dans lui-même par:

$$
\Lambda^{s} f=L^{-1}\left((-\mathrm{i} \omega)^{s} \hat{f}(\omega)\right) \text {. }
$$

Définition, bien entendu, consistante avec la condition $(\boldsymbol{L})$. 
E étant dorénavant hilbertien, la formule de Parseval donne l'équivalence:

$$
f \in H_{\sigma}^{s}\left(\mathbf{R}_{+}, E\right) \Leftrightarrow\left\{\begin{array}{l}
f \text { vérifie }(L) \text { et } \\
\int_{-\infty+\mathrm{i} \sigma}^{+\infty+\mathrm{i} \sigma}|\omega|^{2 s}\|\hat{f}(\omega)\|_{E}^{2} \mathrm{~d} \omega<\infty
\end{array} .\right.
$$

Et on notera indistinctement (quant il n'est pas nécessaire de les distinguer) les deux normes proportionnelles:

$$
f \rightarrow\left(\int_{-\infty}^{\infty} \mathrm{e}^{-2 \sigma t}\left\|\Lambda^{s} f(t)\right\|_{E}^{2} \mathrm{~d} t\right)^{1 / 2}
$$

et

$$
f \rightarrow\left(\int_{-\infty}^{+\infty}\left(\eta^{2}+\sigma^{2}\right)\|\hat{f}(\eta+\mathrm{i} \sigma)\|_{E}^{2} \mathrm{~d} \eta\right)^{1 / 2}
$$

par $\|f\|_{\sigma, s, E}$. Dans les cas qui nous intéressent, $E$ sera surtout de type $H^{r}(\Gamma)$, on écrira alors $|f|_{\sigma, s, r}$ pour $\|f\|_{\sigma, s, H^{r}(\Pi)}$.

Remarquons que pour $s=k$ entier, $A^{s} n^{\prime}$ est autre que la dérivée $k^{e}$ en $t$, et

$$
H_{\sigma}^{k}\left(\mathbf{R}_{+}, E\right)=\left\{f \in L^{\prime}(E) ; \mathrm{e}^{-\sigma t} f^{(k)}(t) \in L^{2}\left(\mathbf{R}_{t}, E\right)\right\} .
$$

\subsection{Résultats d'existence et d'unicité}

Par la formule de Parseval, nous pouvons traduire immédiatement les propositions 1 et 2 en:

Théorème 2. a) Sous l'hypothèse

$$
g \in H_{\sigma_{0}}^{3 / 2}\left(\mathbf{R}_{+}, H^{1 / 2}(\Gamma)\right)
$$

pour un $\sigma_{0}$ (que l'on peut toujours supposer $>0$ ), le problème $\left(P_{+}\right)$admet une solution unique dans $H_{\sigma_{0}}^{0}\left(\mathbf{R}_{+}, H^{1}\left(\Omega_{+}\right)\right)$vérifiant l'inégalité d'énergie:

$$
\left\{\begin{array}{l}
\int_{-\infty}^{+\infty} \mathrm{e}^{-2 \sigma t} E_{+}(t) \mathrm{d} t \leqslant C \frac{1}{\sigma^{2}} \max \left(\frac{1}{\sigma_{0}}, 1\right)|g|_{\sigma, 3 / 2,1 / 2}^{2} \\
\forall \sigma \geqslant \sigma_{0}>0
\end{array}\right.
$$

où $C$ est une constante ne dépendant que de $\Gamma$, et

$$
E_{+}(t)=\int_{\Omega_{+}}\left(|\nabla u(t, x)|^{2}+\left|\frac{\partial u}{\partial t}(t, x)\right|^{2}\right) .
$$

b) Le même résultat est valable pour le problème intérieur $\left(P_{-}\right)$correspondant, la solution u de $\left(P_{+}\right) \cup\left(P_{-}\right)$est alors représentable d'une façon unique comme un 
potentiel retardé de simple couche sur $\Gamma$. La densité $\varphi$ de ce potentiel, définie par $\varphi=[\partial u / \partial \nu]$, satisfait $\grave{a}$ :

$$
\left\{\begin{array}{l}
|\varphi|_{\sigma,-1 / 2,-1 / 2} \leqslant C \frac{1}{\sigma} \max \left(\frac{1}{\sigma_{0}}, 1\right)|g|_{\sigma, 3 / 2,1 / 2} \\
\forall \sigma \geqslant \sigma_{0}>0 .
\end{array}\right.
$$

Remarque 1. Le résultat du th. 2a) est assez différent de celui donné dans Lions-Magenes [15], th. 6.1 du chapitre 5 (voir l'exemple 6.2). Nous n'avons en fait utilisé aucun argument de dualité en temps. Par ailleurs, Lions-Magenes signalent que aucun résultat de régularité qu'ils donnent dans ce chapitre 5 n'était optimal. Dans le cadre fonctionnel adopté ici, ce problème d'optimalité correspond à la question: "est-il possible d'utiliser une puissance plus petite que $3 / 2$ dans l'hypothése (3.2)?". La méthode du $\$ 2$ ne permet pas en tout cas de l'améliorer. On peut aussi choisir un cadre fonctionnel où le temps et l'espace restent couplés. Nous les avons découplés ici pour plus de facilités dans la partie discrétisation.

Remarque 2 . On peut déduire aussi de l'inégalité (2.7) un résultat sur le domaine de dépendance de $\varphi$ en fonction de $g$ : si supp $g \subset t t \geqslant t_{0} ;$, il en est de même pour $\varphi$. Ceci, en utilisant le fait que cette propriété de support se traduit avec la transformée de Laplace de $g$ par l'inégalité:

$$
|\hat{g}(\omega)| \leqslant C \mathrm{e}^{-\sigma t_{0}}(1+|\omega|)^{k}
$$

pour tout $\omega=\eta+\mathrm{i} \sigma$ dans un demi-plan $\left\{\sigma \geqslant \sigma_{1}>\sigma_{0}\right\}$. Le calcul de $\varphi(t, \cdot)$ ne fait intervenir donc que les valeurs de $g(s), s \leqslant t$. C'est ce qu'on revérifiera dans le $\$ 4$.

Par contre, il n'est évidemment pas question de prouver que si suppg $C\{0 \leqslant t \leqslant T\}$, il en sera de même pour $\varphi$ : des réflexions sur $\Gamma$ peuvent subsister après le passage de l'onde incidente. L'extension des calculs à $\mathbf{R}_{+}$tout entier est donc nécessaire même si $g$ est donné avec supp $g \subset[0, T]$.

Remarque 3. Dans le cas d'un demi-espace, et dans un cadre $L^{2}\left(0, T ; H^{0}(I)\right)$, Lebeau-Schatzman [14] démontre la propriété de positivité suivante:

$$
\left.\int_{0}^{T}\left\langle N_{+} g, g\right\rangle \mathrm{d} t\right\rangle 0
$$

pour l'opérateur de Neumann $N_{+} g=(\partial u / \partial v)_{+}, u$ étant la solution $d u$ problème $\left(P_{+}\right)$(avec $\left.\Omega_{+}=\left\{x_{3}>0\right\}\right)$. Ils montrent aussi que cette positivité n'est plus vraie pour $\Omega_{+}$quelconque. Nous avons à cet égard le résultat suivant qui traduit (2.8):

Proposition 4. L'opérateur $N_{+}$est coercif au sens de l'inégalité:

$$
\int_{-\infty}^{\infty} \mathrm{e}^{-2 \sigma t}\left\langle-N_{+} g, \frac{\partial g}{\partial t}\right\rangle \mathrm{d} t \geqslant C \int_{-\infty}^{\infty} \mathrm{e}^{-2 \sigma t}|g(t)|_{1 / 2}^{2} \mathrm{~d} t
$$

pour rout $\sigma \geqslant \sigma_{0}>0, C$ étant une constante ne dépendant que de $\Gamma$ et $\sigma_{0}$. 


\subsection{Formulation variationnelle pour le calcul de $\varphi$}

Il nous reste à traduire la proposition 3 pour obtenir une propriété de coercivité pour l'opérateur du potentiel retardé:

$$
S \varphi(t, x)=\frac{1}{4 \pi} \int_{\Gamma} \frac{\varphi(t-|x-y|, y)}{|x-y|} \mathrm{d} \sigma(y) \quad(t>0, x \in \Gamma) .
$$

Comme $-\mathrm{i} \omega S_{\omega} \hat{\varphi}(\omega, \cdot)$ est la transformée de $\frac{\partial}{\partial t}(S \varphi)=S\left(\frac{\partial \varphi}{\partial t}\right)$, et la formule de Parseval établit une égalité entre les intégrales

$$
\frac{1}{2 \pi} \int_{-\infty+i \sigma}^{+\infty+i \sigma}\left\langle\hat{\varphi}(\omega),-i \omega S_{\omega} \hat{\varphi}(\omega)\right\rangle \mathrm{d} \omega
$$

et

$$
\int_{-\infty}^{+\infty} \mathrm{e}^{-2 \sigma t}\left\langle\varphi(t), \frac{\partial}{\partial t} S \varphi(t)\right\rangle \mathrm{d} t
$$

On peut formuler le

Théorème 3. a) $S$ est un opérateur linéaire continu de $H_{\sigma}^{1}\left(\boldsymbol{R}_{+}, H^{-1 / 2}(\Gamma)\right)$ dans $H_{\sigma}^{0}\left(\mathbf{R}_{+}, H^{+1 / 2}(\Gamma)\right)$ de norme bornée indépendamment de $\sigma \geqslant \sigma_{0}>0$. Plus précisément:

$$
\int_{-\infty}^{\infty} \mathrm{e}^{-2 \sigma t}|S \varphi(t)|_{1 / 2}^{2} \mathrm{~d} t \leqslant C \frac{1}{\sigma_{0}^{2}} \max \left(\frac{1}{\sigma_{0}^{4}}, 1\right) \int_{-\infty}^{+\infty} \mathrm{e}^{-2 \sigma t}\left|\varphi^{\prime}(t)\right|_{-1 / 2}^{2} \mathrm{~d} t .
$$

b) Pour $\varphi$ appartenant à:

$$
D(S)=\left\{\varphi \in H_{\sigma}^{1}\left(\mathbf{R}_{+}, H^{-1 / 2}(\Gamma)\right) \text { tel que } S \varphi \in H_{\sigma}^{1}\left(\mathbf{R}_{+}, H^{1 / 2}(\Gamma)\right\}\right.
$$

on a l'inégalité de coercivité

$$
\int_{-\infty}^{+\infty} \mathrm{e}^{-2 \sigma t}\left\langle\varphi(t), S \varphi^{\prime}(t)\right\rangle \mathrm{d} t \geqslant C \min \left(\sigma_{0}, 1\right)|\varphi|_{\sigma,-1 / 2,-1 / 2}^{2} .
$$

Ce sont en effet les équivalences des formules (2.10) et (2.11).

Ainsi, s'il semble normal que dans le passage $g \rightarrow \varphi=N g=[\partial u / \partial v]$, on perd de régularité en temps et en espace, dans le passage inverse $\varphi \rightarrow g=S \varphi$, on a regagné la régularité en espace mais perd toujours un degré de régularité en temps. Nous ne sommes pas arrivés à surmonter cette contradiction. Néanmoins, le résultat obtenu va donner quand même une méthode d'approximation stable et convergente du problème de calcul de $\varphi$ à partir de $g$.

Pour cela, on remplace l'équation du potentiel retardé

$$
S \varphi=g
$$

par l'équation variationnelle: 


$$
\left\{\begin{array}{l}
\left.\int_{-\infty}^{+\infty} \mathrm{e}^{-2 \sigma t}\left(\iint_{\Gamma \times \Gamma} \frac{\psi(t, x) \varphi_{t}^{\prime}(t-|x-y|, y)}{4 \pi|x-y|} \mathrm{d} \sigma_{x} \mathrm{~d} \sigma_{y}\right)\right) \mathrm{d} t \\
=\int_{-\infty}^{\infty} \mathrm{e}^{-2 \sigma t} \int_{\Gamma} \psi(t, x) g_{t}^{\prime}(t, x) \mathrm{d} \sigma_{x} \mathrm{~d} \sigma_{y} \quad \forall \psi \in H_{\sigma}^{1}\left(\mathbf{R}_{+}, H^{-1 / 2}(\Gamma)\right) .
\end{array}\right.
$$

Si $\varphi$ est dans $D(S)$, elle vérifie (3.6). Inversement, à partir de (3.6), on va montrer qu'un problème discrétisé donne une solution unique $\varphi_{h, \Delta t}$ qui approche bien $p$ dans la norme de $H_{\sigma}^{-1 / 2}\left(\mathrm{R}_{+}, H^{-1 / 2}(\Gamma)\right)$.

\section{\$4 Dicrétisation: les schemas}

\subsection{Dicrétisation en espace}

Nous suivons Nédelec [11]. La surface $(\Gamma)$ sera supposée découpée en $p$ morceaux fermés $\left(\Gamma_{i}\right)$, tels que

(a) $\bigcup_{1}^{p} \Gamma_{i}=\Gamma$

(b) $\Gamma_{i} \cap \Gamma_{j}$ est vide ou est une courbe portée par $\Gamma$.

(c) Pour tout $i, \Gamma_{i}$ est l'image biunivoque par une application régulière $\chi_{i}$ d'un domaine polyhédral $D_{i}$ dans $R^{2}$.

Chacun des domaines $D_{i}$ sera alors partagé par une «triangulation régulière» $T_{h i}$ en éléments $K$. En se donnant un élément fini (lagrangien) de classe $C^{0}(K, \Sigma, \mathrm{P})$ sur $K$, on construit ensuite l'élément de surface $K_{h}$ image de $K$ par l'interpolé $\chi_{i}$ par l'élément fini ainsi considéré. La surface $\left(\Gamma_{h}\right)$ sera ensuite la réunion des $K_{h}$. Le cas le plus simple est évidemment celui de l'élément fini $P_{1}$, où $\Gamma_{h}$ est une réunion de facettes triangulaires planes dont les sommets sont sur $(I)$.

De façon générale, fixons l'entier $m_{0} \geqslant 1$ tel que $\mathbf{P}_{m_{0}} \subset \mathbf{P}, \mathbf{P}_{m_{0}}$ étant les polynômes de degré $\leqslant m_{0}$ sur $R^{2}$. Dans tous les cas, pour $h$ suffisamment petit, $\left(\Gamma_{h}\right)$ se trouvera dans un voisinage tubulaire $\left.\Gamma_{\delta}=\Gamma \times\right]-\delta,+\delta[$ de $\Gamma$, et l'application projection orthogonale $P$ sur $\Gamma$, définie dans ce voisinage, sera une bijection si restreinte à $\Gamma_{h}$. Nous désignons alors par $P^{-1}$ l'inverse de $\left(\left.P\right|_{\Gamma_{h}}: \Gamma_{h} \rightarrow \Gamma\right)$. Une fonction $\varphi$ définie sur $\Gamma_{h}$ aura une image naturelle sur $\Gamma$ définie par $\tilde{\varphi}=\varphi_{0} P^{-1}$. Nous utiliserons souvent cette correspondance entre fonctions sur $\Gamma_{h}$ et fonctions sur $\Gamma$.

D'autre part, nous définissons un espace $V_{h}$ de fonctions définies sur $\Gamma_{h}$ à partir d'un élément fini $\left(K, \Sigma^{*}, P^{*}\right)$ tel que $P^{*} \supset \mathrm{P}_{m_{1}}$. L'espace des fonctions $\left\{\tilde{\varphi}=\varphi_{0} P^{-1} ; \varphi \in V_{h}\right\}$ sera noté $\bar{V}_{h}$. Comme on a besoin d'approcher $H^{-1 / 2}(\Gamma)$, le choix le plus simple peut être fait avec $m_{1}=0$. La discrétisation en espace peut maintenant se faire, soit en gardant la surface exacte, soit en la remplaçant par la surface approchée $\Gamma_{h}$.

Dans le ler cas, on pose le problème approché suivant: 


$$
\left\{\begin{array}{l}
\text { chercher } \varphi_{h} \in H_{\sigma}^{1}\left(\mathbf{R}_{+}, \tilde{V}_{h}\right) \text { telle que } \\
\int_{0}^{\infty} \mathrm{e}^{-2 \sigma t} \int_{\Gamma} \frac{\psi_{h}(t, x) \varphi_{h}^{\prime}(t-|x-y|, y)}{4 \pi|x-y|} \mathrm{d} \sigma_{x} \mathrm{~d} \sigma_{y} \mathrm{~d} t \\
=\int_{0}^{\infty} \mathrm{e}^{-2 \omega t} \int_{\Gamma} \psi_{h}(t, x) g_{h}^{\prime}(t, x) \mathrm{d} \sigma_{x} \mathrm{~d} t \\
\text { pour tout } \psi_{h} \in H_{\sigma}^{1}\left(\mathbf{R}_{+}, \tilde{V}_{h}\right)
\end{array}\right.
$$

où $g_{h}^{\prime}$ est une approximation de $g^{\prime}$.

Dans le $2^{\mathrm{d}}$ cas, il suffit de remplacer $\tilde{V}_{h}$ par $V_{h}$ et $\Gamma$ par $\Gamma_{h}$. Nous explicitons ci-dessous seulement le problème (4.1). Soit $\left(\varphi_{h}^{i}\right)_{1 \leqslant j \leqslant N_{h}}$ une base de $\tilde{V}_{h}$. Les éléments de $H_{\sigma}^{1}\left(\boldsymbol{R}_{+}, \tilde{V}_{h}\right)$ peuvent s'écrire:

$$
\psi_{h}(t, x)=\sum_{j=1}^{N_{h}} \beta_{j}(t) \varphi_{h}^{j}(x)
$$

avec

$$
\beta_{j} \in H_{\sigma}^{1}\left(\mathbf{R}_{+}, \mathbf{R}\right) \quad \forall j=1, \ldots, N_{h}
$$

On obtient alors la formulation suivante de (4.1)

$$
\text { (4.2) }\left\{\begin{array}{l}
\text { chercher } \alpha_{l} \in H_{\sigma}^{1}\left(\mathbf{R}_{+}, \mathbf{R}\right), l=1, \ldots, N_{h} \text { telles que: } \\
\sum_{l} \int_{0}^{\infty} \mathrm{e}^{-2 \sigma t} \beta_{j}(t) \iint_{\Gamma \times \Gamma} \frac{\varphi_{h}^{j}(x) \varphi_{h}^{\prime}(y)}{4 \pi|x-y|} \alpha_{l}^{\prime}(t-|x-y|) \mathrm{d} \sigma_{x} \mathrm{~d} \sigma_{y} \mathrm{~d} t \\
=\int_{0}^{\infty} \mathrm{e}^{-2 \sigma t} \beta_{j}(t) \int_{\Gamma} \varphi_{h}^{j}(x) g_{h}^{\prime}(t, x) \mathrm{d} \sigma_{x} \quad \forall j, \beta_{j} \in H_{\sigma}^{1}\left(\mathbf{R}_{+}, \mathbf{R}\right) .
\end{array}\right.
$$

\subsection{Discrétisation en temps}

Soient maintenant $\Delta t>0$ et $\left\{t_{n}=n \Delta t ; n \in \mathbf{N}\right\}$ une subdivision de $\mathbf{R}_{+}$. On discrétisera le système (4.2) en remplaçant les fonctions $\alpha_{j}$ et $\beta_{j}$ par $\alpha_{j, \Delta t}$ et $\beta_{j, \Delta t}$ polynômiales de degré $m_{2}$ dans chaque intervalle $I_{m}=\left(t_{n}, t_{n+1}\right)$. Pour rester dans $H_{\sigma}^{1}\left(\mathbf{R}_{+}, \mathbf{R}\right)$, le plus petit degré $m_{2}$ qui convient est $m_{2}=1$, chaque fonction $\alpha_{j}$ est alors affine par morceaux, continue (et nulle en 0 ). Nous commençons par démontrer que ce faisant, on obtient un schéma calculable pour les $\alpha_{j, \Delta t}$. Le cas $m_{2}>1$ sera fait au numéro suivant.

Nous prenons donc

$$
\alpha_{j, \Delta t}(t)=\int_{0}^{t} \alpha_{j, \Delta t}(s) \mathrm{d} s
$$

avec

$$
\alpha_{j, \Delta t}^{\prime}(t)=a_{j}^{n} \text { pour } t \in I_{n} .
$$

Il est immédiat que:

(4.3) $\alpha_{j, \Delta t}=\Delta t \sum_{k=0}^{n-1} \dot{a}_{j}^{k}+a_{j}^{n}\left(t-t_{n}\right) \quad$ pour $t \in I_{n}$. 
Ecrivons maintenant le système (4.2) avec $\alpha_{j, \Delta t}$ et $\left(\beta_{j, \Delta t}^{n}\right)_{n \in N}$ de même forme (4.3) servant de fonctions de base:

$$
\beta_{j, \Delta t}^{n}=\beta_{\Delta t}^{n}(t)= \begin{cases}0 & t<t_{n} \\ t-t_{n} & t_{n} \leqslant t \leqslant t_{n+1} . \\ \Delta t & t>t_{n+1}\end{cases}
$$

Plus exactement, on ecrit avec $\beta_{\Delta t}^{n}$ et $\beta_{\Delta t}^{n-1}$ et fait la soustraction:

$$
\left\{\begin{array}{l}
\sum_{l}\left(\int_{t_{n}}^{t_{n+1}} \mathrm{e}^{-2 \sigma t}\left(t-t_{n}-\Delta t\right) \iint_{\Gamma \times \Gamma} \frac{\varphi_{h}^{j}(x) \varphi_{h}^{l}(y)}{\pi|x-y|} \alpha_{l, \Delta t}^{\prime}(t-|x-y|) \mathrm{d} \sigma_{x} \mathrm{~d} \sigma_{y} \mathrm{~d} t\right. \\
\left.\quad-\int_{t_{n-1}}^{t_{n}} \mathrm{e}^{-2 \sigma t}\left(t-t_{n-1}\right) \iint_{\Gamma \times \Gamma} \frac{\varphi_{h}^{\prime}(x) \varphi_{h}^{\prime}(y)}{4 \pi|x-y|} \alpha_{l, \Delta t}^{\prime}(t-|x-y|) \mathrm{d} \sigma_{x} \mathrm{~d} \sigma_{y} \mathrm{~d} t\right) \\
=\left(\int_{t_{n}}^{t_{n+1}} \mathrm{e}^{-2 \sigma t}\left(t-t_{n}-\Delta t\right) \iint_{\Gamma}^{j}(x) g_{h}^{\prime}(t, x) \mathrm{d} \sigma_{x} \mathrm{~d} t\right. \\
\left.\quad-\int_{t_{n-1}}^{t_{n}} \mathrm{e}^{-2 \sigma t}\left(t-t_{n-1}\right) \int \varphi_{\Gamma} \varphi_{h}^{j}(x) g_{h}^{\prime}(t, x) \mathrm{d} \sigma_{x} \mathrm{~d} t\right) \\
\forall j=1, \ldots, N_{h}, n \geqslant 1 \text { (pour } n=0, \text { les termes en } \int_{t_{n-1}}^{t_{n}} \text { disparaissent). }
\end{array}\right.
$$

C'est un système linéaire des vecteurs

$$
A^{k}=\left(a^{k}, \ldots, a_{N_{h}}^{k}\right)^{t} \quad 0 \leqslant k \leqslant n
$$

dont on va démontrer la résolubilité de proche en proche.

Proposition 5. Le schéma (4.4) est constructif. Plus précisément, il peut s'écrire sous la forme:

$$
\text { (4.5) } \sum_{k=0}^{n} M^{n-k} A^{k}=B^{n}
$$

ou la matrice $M^{0}$ est symétrique, inversible pour $\Delta t$ petit, et les matrices $M^{n-k}$ nulles si $n-k>[d(I) / \Delta t]$, ou $[x]$ est la partie entiere de $x$.

Démonstration. Le membre de gauche de (4.4) peut en effet s'écrire:

$$
G=\sum_{k=0}^{n} M^{n, k} A^{k}
$$

avec les matrices $M^{n, k}$ données par:

$$
\begin{aligned}
M_{j, l}^{n, n} & =\int_{t_{n}}^{t_{n+1}} \mathrm{e}^{-2 \sigma t}\left(t-t_{n+1}\right) \iiint_{\left\{|x-y| \leqslant t-t_{n}\right\}} \frac{\varphi_{h}^{j}(x) \varphi_{h}^{l}(y)}{4 \pi|x-y|} \mathrm{d} \sigma_{x} \mathrm{~d} \sigma_{y} \mathrm{~d} t \\
& =\mathrm{e}^{2 \sigma t_{n}} \int_{0}^{\Delta t} \mathrm{e}^{-2 \sigma s}(s-\Delta t) \iiint_{\{|x-y| \leqslant s\}} \frac{\varphi_{h}^{j}(x) \varphi_{h}^{l}(y)}{\pi|x-y|} \mathrm{d} \sigma_{x} \mathrm{~d} \sigma_{y} \mathrm{~d} t
\end{aligned}
$$


et de même pour $k<n$ :

$$
M_{j, l}^{n, k}=\mathrm{e}^{-2 \sigma t_{n}} \int_{0}^{\Delta l} \mathrm{e}^{-2 \sigma s}\left[(s-\Delta t) f_{j, l}^{n-k}(s)-s \cdot \mathrm{e}^{-2 \sigma \Delta s} f_{j, l}^{n-k}(s-\Delta s)\right] \mathrm{d} s
$$

où l'on a posé

$$
f_{j, l}^{n-k}(s)=\iint \frac{\varphi_{h}^{j}(x) \varphi_{h}^{l}(y)}{4 \pi|x-y|} \mathrm{d} \sigma_{x} \mathrm{~d} \sigma_{y}\left\{s-\Delta s+t_{n-k}<|x-y| \leqslant s+t_{n-k}\right\} .
$$

Il est aussi clair que $e^{-2 \sigma t_{n}}$ peut être mis en facteur du second membre de (4.4), avec un facteur indépendant de $n$. En changeant de signe, on a la formule (4.5) avec la matrice $M^{0}$ donnée par:

$$
M_{j, l}^{0}=\int_{0}^{\Delta t} \mathrm{e}^{-2 \sigma s}(\Delta t-s) \iint \frac{\phi_{h}^{j}(x) \varphi_{h}^{l}(y)}{4 \pi|x-y|} \mathrm{d} \sigma_{x} \mathrm{~d} \sigma_{y} \mathrm{~d} t \quad\{|x-y| \leqslant s\} .
$$

Il est évident qu'elle est symétrique. Dans l'intervalle d'intégration $(0, \Delta t)$, on peut assimiler l'ensemble $\{y ;|x-y| \leqslant s\}$ au disque de centre 0 , de rayon $s$ situé dans le plan tangent en $x$ à $\Gamma$, d'où :

$$
\int \frac{\varphi_{h}^{l}(y) \mathrm{d} \sigma_{y}}{|x-y|}=2 \pi \varphi_{h}^{l}(x) \cdot s+o(\Delta t) \quad(|x-y| \leqslant s)
$$

par suite:

$$
\left\{\begin{aligned}
M_{j, l}^{0} & =\frac{1}{2}\left(\int_{\Gamma} \varphi_{h}^{j}(x) \cdot \varphi_{h}^{l}(x) \mathrm{d} \sigma_{x}\right)\left(\int_{0}^{\Delta t} \mathrm{e}^{-2 \sigma s} s \cdot(\Delta t-s) \mathrm{d} s\right)(1+o(\Delta t)) \\
& =\frac{\Delta t^{3}}{6}\left(\int_{\Gamma} \varphi_{h}^{j}(x) \varphi_{h}^{l}(x) \mathrm{d} \sigma_{x}\right)(1+o(\Delta t)) .
\end{aligned}\right.
$$

Donc à un terme multiplicatif près, $M^{0}$ n'est autre que la matrice des produits scalaires des fonctions $\left(\varphi_{h}^{j}\right)$ qui sont linéairement indépendants. Elle est non seulement inversible, mais "facile à inverser", et de plus creuse si les supports des $\varphi_{h}^{j}$ sont petits, ce qui est le cas dans la méthode des éléments finis.

Enfin, la nullité des termes $M^{n-k}$ pour $n-k>[d(\Gamma) / t]$ est évidente.

La généralisation du schéma (4.4) au cas $m_{2}>1$ est chose facile. Posant $\alpha_{l, \Delta t}^{\left(m_{2}\right)}(t)=a_{l}^{n}$ pour $t \in I_{n}$, on remarquera encore que $\alpha_{l, \Delta t}^{\prime}(t)$ s'écrit

$$
\left\{\alpha_{l, \Delta t}^{\prime}(t)=\frac{a_{l}^{n}}{\left(m_{2}-1\right) !}\left(t-t_{n}\right)^{m_{2}-1}+\left\{\text { termes de degré } m_{2}-1\right\} \text { pour } t \in I_{n}\right.
$$

ou le crochet \{termes de degré $\left.<m_{2}-1\right\}$ ne fait intervenir que les $\left(a_{l}^{p}\right)$ pour $0 \leqslant p \leqslant n-1$. On obtient alors le schéma voulu en appliquant (4.2) avec $\beta_{j, \Delta t}$ égale à une combinaison convenable de fonctions de la base canonique de $\boldsymbol{H}_{\sigma}^{m_{2}}(\Delta t ; \mathbf{R})$, le sous-espace de $\boldsymbol{H}_{\sigma}^{m_{2}}\left(\mathbf{R}_{+}, \mathbf{R}\right)$ composée de fonctions dont la dérivée $m_{2}^{e}$ est constante dans chaque intervalle $I_{n}$. 
Par exemple, pour $m_{2}=2$, cette base canonique sera:

$$
\beta_{\Delta t}^{n}(t)= \begin{cases}0 & t \leqslant t_{n} \\ \frac{1}{2}\left(t-t_{n}\right)^{2} & t_{n} \leqslant t \leqslant t_{n+1} \\ \frac{\Delta t^{2}}{2}+\Delta t\left(t-t_{n+1}\right) & t \geqslant t_{n+1}\end{cases}
$$

on obtient alors le système analogue à (5) avec

$$
\beta_{j, \Delta t}=\beta_{\Delta t}^{n-2}-2 \beta_{\Delta t}^{n-1}+\beta_{\Delta t}^{n} \quad(n \geqslant 0)
$$

qui est nulle en dehors de l'intervalle $\left[t_{n-2}, t_{n+1}\right]$. Le système obtenu est encore un système de la forme (4.5), où maintenant $A^{k}$ est le vecteur des valeurs $\alpha_{j, \Delta r}^{\prime \prime}$ dans l'intervalle $I_{K}$.

\section{\$5 Analyse du schéma. Cas de la surface exacte}

Nous donnons dans ce paragraphe un résultat de stabilité du schéma proposé (th. 4) et quelques estimations d'erreurs (th. 5, 6,7). Les numéros 5.1 et 5.2 sont des résultats techniques dans les espaces considérés.

\subsection{Inégalités dans $H_{\sigma}^{m}(\Delta t ; \mathrm{R})$}

On a vu au $\S 4$ que la solution approchée $\varphi_{h, \Delta t}=\sum_{j=1}^{N} \alpha_{j, \Delta t}(t) \varphi_{h}^{j}$ est cherchée dans les espaces $H_{\sigma}^{m}\left(\Delta t ; \tilde{V}_{h}\right)$ composés de fonctions en $t$, à valeurs dans $\tilde{V}_{h} \simeq \mathbf{R}^{N}$, dont la dérivée d'ordre $m$ est constante dans chaque intervalle $(n \Delta t,(n+1) \Delta t)=I_{n}$.

Si $f \in H_{\sigma}^{m}(\Delta t ; \mathbf{R})$, toutes ses "primitives" $\Lambda^{-s} f(s \geqslant 0)$ sont dans $H_{\sigma}^{0}(\Delta t ; \mathbf{R})$. On commence par donner dans ce numéro quelques inégalités concernant les normes dans ce dernier espace des $\Lambda^{-s} f$.

Nous avons noté $\|f\|_{\sigma, s, E}$ pour la norme dans $H_{\sigma}^{s}\left(R_{+}, E\right)$. Pour $E=\mathbf{R}$, on écrira simplement $\|f\|_{\sigma, s}$ à la place de $\|f\|_{\sigma, s, \mathrm{R}}$.

Lemme 2. Pour $\sigma_{0}>0, \Delta t_{0}>0$ et $k$ entier $\geqslant 1$, il existe une constante $c>0$ telle que

(5.1) $|f|_{\sigma,(-k+1)} \leqslant C \Delta t^{-1}|f|_{\sigma,-k}$

pour tout $f \in H_{\sigma}^{0}(\Delta t ; \mathbf{R}), 0 \leqslant \sigma \leqslant \sigma_{0}$ et $0<\Delta t \leqslant \Delta t_{0}$.

Démonstration. On part de l'expression de la primitive d'ordre $k \operatorname{de} f$ :

$$
\Lambda^{-k}(t)=\sum_{l=0}^{k} \alpha_{k, l}^{n}\left(t-t_{n}\right)^{l} \quad \text { pour } t \in I_{n}
$$

avec

$$
\left\{\begin{array}{l}
\alpha_{k, l}^{n}=\Delta t^{k-l} L_{k, l}^{n}\left(a_{0}, \ldots, a_{n}\right) \\
0 \leqslant l \leqslant k
\end{array}\right.
$$


si $a_{n}=f(t)$ dans $I_{n}, L_{l, k}^{n}$ est une forme linéaire des $a_{0}, \ldots, a_{n}$. La vérification par récurrence de (5.2) est immédiate.

Alors

$$
\Lambda^{-(k-1)} f(t)=\sum_{=1}^{k} \alpha_{k, l}^{n} l\left(t-t_{n}\right)^{l-1} \quad\left(t \in I_{n}\right)
$$

Il s'agit donc de comparer:

$$
|f|_{\sigma,-k}^{2}=\left|\Lambda^{-k} f\right|_{\sigma, 0}=\sum_{n \geqslant 0} \int_{t_{n}}^{t_{n+1}} \mathrm{e}^{-2 \sigma t}\left(\sum_{l=0}^{k} \alpha_{k, l}^{n}\left(t-t_{n}\right)^{l}\right)^{2} \mathrm{~d} t
$$

et

$$
|f|_{\sigma,-k+1}^{2}=\sum_{n \geqslant 0} \int_{t_{n}}^{t_{n+1}} e^{-2 \sigma t}\left(\sum_{l=1}^{k} l \alpha_{k, l}^{n}\left(t-t_{n}\right)^{l-1}\right)^{2} \mathrm{~d} t .
$$

Posons:

$$
\begin{aligned}
A_{n} & =\int_{t_{n}}^{t_{n+1}} \mathrm{e}^{-2 \sigma t}\left(\sum_{l=0}^{k} \alpha_{l}\left(t-t_{n}\right)^{l}\right)^{2} \mathrm{~d} t=\mathrm{e}^{-2 \sigma t_{n}} \int_{0}^{\Delta t} \mathrm{e}^{-2 \sigma s}\left(\sum_{l=0}^{k} \alpha_{l} s^{\prime}\right)^{2} \mathrm{~d} s \\
& =\mathrm{e}^{-2 \sigma t_{n} \Delta t^{2 k+1}} \int_{0}^{1} \mathrm{e}^{-2 \sigma \Delta t \cdot s}\left(\sum_{l=0}^{k} \alpha_{i} s^{l}\right)^{2} \mathrm{~d} s
\end{aligned}
$$

où $\alpha_{l}=\Delta t^{k-l} \alpha_{l}^{\prime} ;$ puis

$$
\begin{aligned}
B_{n} & =\int_{t_{n}}^{t_{n+1}} \mathrm{e}^{-2 \sigma t}\left(\sum_{l=1}^{k} l \alpha_{l}\left(t-t_{n}\right)^{l-1}\right)^{2} \mathrm{~d} t \\
& =\mathrm{e}^{-2 \sigma t_{n}} \Delta t^{2 k-1} \int_{0}^{1} \mathrm{e}^{-2 \sigma \Delta t \cdot s}\left(\sum_{l=1}^{k} l \alpha_{l} s^{l-1}\right)^{2} \mathrm{~d} s
\end{aligned}
$$

comme il est clair que la forme quadratique

$$
q_{A}\left(\alpha^{\prime}\right)=\int_{0}^{1} \mathrm{e}^{-\mu s}\left(\sum_{l=0}^{k} \alpha_{l}^{\prime} s^{l}\right)^{2} \mathrm{~d} s
$$

représente le carré d'une norme sur $\mathrm{R}^{k+1}$ de $\alpha^{\prime}$, uniformément équivalente à la norme euclidienne pour $0 \leqslant \mu \leqslant \mu_{0}$, on a

$$
\Delta t^{2} \cdot B_{n} \leqslant C A_{n}
$$

où la constante $C$ ne dépend que de $k$ et $\mu_{0}$, pour tous les $\sigma, \Delta t$ tels que $2 \sigma \cdot \Delta t$ $\leqslant \mu_{0}$ et tous les vecteurs $\alpha^{\prime} \in \mathbf{R}^{k+1}$. D'où immédiatement le résultat.

Corollaire. Pour tout $s>0$, il existe $\Delta t_{0}>0$ et $c>0$ telles que

(5.3) $\Delta t^{s}|f|_{\sigma, 0} \leqslant C|f|_{\sigma,-s}$

pour tout $f \in H_{\sigma}^{m}(\Delta t ; \mathbf{R}), 0<\Delta t<\Delta t_{0}$. 
On a en effet $|f|_{\sigma,-s}=\left|\Lambda^{-s} f\right|_{\sigma, 0}$ et pour $s$ entier, (5.1) permet de conclure. Pour $s>0$, non entier, soit $k<s<k+1$, on écrit $g=\Lambda^{-(k+1)} f \in H_{\sigma}^{m+k+1}(\Delta t ; R)$ et

$$
|g|_{\sigma, k} \leqslant C_{1} \Delta t^{-k}|g|_{\sigma, 0}, \quad|g|_{\sigma, k+1} \leqslant C_{2} \Delta t^{-k-1}|g|_{\sigma, 0}
$$

puis on interpole.

Remarques. a) Les inégalités (5.1 et 3 ) restent valables si l'on remplace $\mathbf{R}$ par un espace hilbertien séparable $H$. Il suffit en effet dans la démonstration cidessus de décomposer les vecteurs $\alpha$ isur une base orthonormale.

b) Dans l'autre sens, il est facile de voir que, pour $\sigma>\sigma_{0}$

$$
|f|_{\sigma,-1} \leqslant \frac{C}{\sigma-\sigma_{0}}|f|_{\sigma_{0}, 0} \quad \forall f \in H_{\sigma}^{0}\left(\mathbf{R}_{+}, \mathbf{R}\right)
$$

mais aucune majoration du ler membre de (5.4) par son second membre multiplié par un facteur $\Delta t^{e}(\varepsilon>0)$ n'est possible, meme si l'on se restreint aux espaces $H_{\sigma}^{k}(\Delta t ; \mathbf{R})$.

\subsection{Interpolation dans $H_{\sigma}^{m}(\Delta t ; \mathrm{R})$}

Nous aurons besoin aussi d'un résultat d'interpolation dans $H_{\sigma}^{m}(\Delta t ; \mathbf{R})$ :

Lemme 3. Soit pour $k<m$, l'opérateur $r_{\Delta t}$ associant d $f \in H_{\sigma}^{m}\left(\mathbf{R}_{+}, \mathbf{R}\right)$ la fonction dans $H_{o}^{k}(\Delta t ; \mathbf{R})$ définie par:

$$
\left\{\begin{array}{l}
\left(r_{\Delta t} f\right)(t)=f\left(t_{n}\right)+\left(t-t_{n}\right) f^{\prime}\left(t_{n}\right)+\cdots+\frac{\left(t-t_{n}\right)^{k}}{k !} f^{(k)}\left(t_{n}\right) \\
\text { pour } t_{n} \leqslant t \leqslant t_{n+1}
\end{array}\right.
$$

on a:

$$
\left|f-r_{\Delta t} f\right|_{\sigma, 0} \leqslant C_{k} \cdot \Delta t^{k+1}|f|_{\sigma, k+1} .
$$

Démonstration. La formule de Taylor avec reste intégrale donne:

$$
\left(f-r_{\Delta t} f\right)(t)=\frac{1}{k !} \int_{t_{n}}^{t}(t-u)^{k} f^{(k+1)}(u) \mathrm{d} u \quad t \in I_{n}
$$

d'où par Cauchy-Schwarz:

$$
\left|f-r_{\Delta t} f\right|^{2} \leqslant \frac{1}{(k !)^{2}(2 k+1)}\left(t-t_{n}\right)^{2 k+1} \int_{t}^{t}\left[f^{(k+1)}(u)\right]^{2} \mathrm{~d} u
$$

par suite:

$$
\begin{aligned}
\int_{t_{n}}^{t_{n+1}} \mathrm{e}^{-2 \sigma t}\left|f(t)-r_{\Delta t} f\right|^{2} \mathrm{~d} t \leqslant & \frac{1}{(2 k+1)(k !)^{2}} \int_{t_{n}}^{t_{n+1}} \mathrm{e}^{-2 \sigma u}\left[f^{(k+1)}(u)\right]^{2} \mathrm{~d} u \\
& \cdot\left(\int_{u}^{t_{n+1}} \mathrm{e}^{-2 \sigma(t-u)}\left(t-t_{n}\right)^{2 k+1} \mathrm{~d} t\right)
\end{aligned}
$$


soit encore

$$
\int_{t_{n}}^{t_{n+1}} \mathrm{e}^{-2 \sigma t}\left|f-r_{\Delta t} f\right|^{2} \mathrm{~d} t \leqslant \frac{\Delta t^{2 k+2}}{k !(2 k+1)(2 k+2)} \int_{t_{n}}^{t_{n+1}} \mathrm{e}^{-2 \sigma u}\left[f^{(k+1)}(u)\right]^{2} \mathrm{~d} u
$$

en sommant sur $n$, on obtient (5.6).

Corollaire. Avec les mêmes notations, on a:

$$
\begin{aligned}
& \left|f-r_{\Delta t} f\right|_{\sigma, 1 / 2} \leqslant C \Delta t^{k+\frac{1}{2}}|f|_{\sigma, k+1} \\
& \left|f-r_{\Delta} f\right|_{\sigma,-1 / 2} \leqslant C \Delta t^{k+\frac{3}{2}}|f|_{\sigma, k+1}
\end{aligned}
$$

- c'est immédiat par interpolation.

Enfin, rappelons les résultats analogues pour la discrétisation en espace.

Lemme 4. Soit $q_{h}$ l'opérateur de projection de $L^{2}(\Gamma)$ sur $\bar{V}_{h}$ le sous-espace construit avec un élément fini d'ordre $m_{1}(\$ 4)$, nous avons si $m_{1}>0$ :

$$
\begin{aligned}
& \left|f-q_{h} f\right|_{L^{2}(I)} \leqslant C h^{m_{1}+1}|f|_{H^{m_{1}+1}(\Gamma)} \\
& \left|f-q_{h} f\right|_{H^{-1 / 2}(\Pi)} \leqslant C h^{m_{1}+{ }_{2}^{3}}|f|_{m_{1}+1}(\Gamma)
\end{aligned}
$$

pour tout $f \in H^{m_{1}+1}(\Gamma)$.

Voir le cours de Nédelec [11], où l'on trouve aussi l'inégalité

(5.11) $|f|_{L^{2}(\Pi)} \leqslant \frac{C}{\sqrt{h}}|f|_{H^{-1 / 2}(\Pi)} \forall f \in \tilde{V}_{h}$

qui nous sera aussi utile dans la suite.

\subsection{Stabilité du schéma}

Soient maintenant $\varphi$ et $\varphi_{h, \Delta t}$ les solutions exactes et calculées de l'équation du potential retardé $S \varphi=g$. La dérivée $\varphi^{\prime}=\partial \varphi / \partial t$ vérifie la relation (3.6), tandis que $f_{h, \Delta t}=\partial \varphi_{h, \Delta t} / \partial t$ a été calculée explicitement comme solutions dans $H_{\sigma}^{m_{2}-1}\left(\Delta t ; \tilde{V}_{h}\right)$ du système approché de (4.2):

$$
\left\{\begin{array}{l}
f_{h, \Delta t} \in H_{0}^{m_{2}-1}\left(\Delta t ; \tilde{V}_{h}\right) \text { telle que } \\
b\left(\psi_{h, \Delta t}, f_{h, \Delta t}\right)=\int_{0}^{\infty} \mathrm{e}^{-2 \sigma t} \int_{\Gamma} \psi_{h, \Delta t}(t, x) g_{h, \Delta t}^{\prime}(t, x) \mathrm{d} \sigma_{x} \mathrm{~d} t \\
\forall \psi_{h, \Delta t} \in H_{\sigma}^{m_{2}}\left(\Delta t ; \tilde{V}_{h}\right)
\end{array}\right.
$$

où la forme $b$ est la triple intégrale:

$$
b\left(\psi_{h, \Delta t}, f_{h, \Delta t}\right)=\int_{0}^{\infty} \mathrm{e}^{-2 \sigma t} \iint_{\Gamma \times \Gamma} \frac{\psi_{h, \Delta t}(t, x) f_{h, \Delta t}(t-|x-y|, y)}{4 \pi|x-y|} \mathrm{d} \sigma_{x} \mathrm{~d} \sigma_{y} \mathrm{~d} t .
$$

Posant pour $\phi_{h, \Delta t} \in H_{\sigma}^{m_{2}-1}\left(\Delta t ; \tilde{V}_{h}\right)$ :

$$
b_{0}\left(\phi_{h, \Delta t}, f_{h, \Delta t}\right)=b\left(\Lambda^{-1} \phi_{h, \Delta l}, f_{h, \Delta t}\right)
$$


le problème (5.12) est un problème variationnel classique avec la forme bilinéaire (non symétrique $b_{0}$ sur $H_{\sigma}^{m_{2}-1}\left(\Delta t ; \tilde{V}_{h}\right)$. D'après (3.5) et (5.3), on a la relation de coercivité suivante, qui rédémontre l'existence et l'unicité de $f_{h, \Delta t}$ dans chacun des sous espaces $H_{\sigma}^{k}\left(\Delta t ; \tilde{V}_{h}\right)$ de $H_{\sigma}^{0}\left(\mathbf{R}_{+}, H^{-1 / 2}(\Gamma)\right)$ :

(5.13) $b_{0}\left(f_{h, \Delta t}, f_{h, \Delta t}\right) \geqslant C\left|\varphi_{h, \Delta t}\right|_{\sigma,-1 / 2,-1 / 2}^{2} \geqslant C \Delta t^{3}\left|f_{h, \Delta t}\right|_{\sigma, 0,-1 / 2}^{2}$.

Nous obtenons évidemment un résultat de stabilité dans ce cadre fonctionnel.

Théorème 4. Si $g_{h, \Delta t}^{\prime}$ est une approximation consistante de $g^{\prime}$ dans $H_{\sigma}^{1 / 2}\left(\mathrm{R}_{+}, H^{1 / 2}(\Gamma)\right)$, le schéma (5.12) est stable au sens suivant:

(5.14) $\left|\varphi_{h, \Delta t}\right|_{\sigma,-1 / 2,-1 / 2} \leqslant$ Const.

quand $h, \Delta t \rightarrow 0$.

Il résulte en effet de (5.13) que

$$
\left|\varphi_{h, \Delta}\right|_{\sigma,-1 / 2,-1 / 2} \leqslant C\left|g_{h, \Delta}^{\prime}\right|_{\sigma, 1 / 2,1 / 2}
$$

d'où le résultat.

Remarques. a) Nous pouvons rapporter ce résultat à l'analyse du $\S 4$. Prenons par exemple $m_{2}=1$. Tenant compte de (5.3), (5.14) implique alors

$$
\Delta t^{3}\left|\varphi_{h, \Delta t}^{\prime}\right|_{\sigma, 0,-1 / 2}^{2} \leqslant \text { Const. }
$$

or, avec les notations du $\S 4$, on a:

$$
\left|\varphi_{h, \Delta t}^{\prime}\right|_{\sigma, 0,-1 / 2}^{2}=\sum_{n \geqslant 0} \int_{t_{n}}^{t_{n+1}} \mathrm{e}^{-2 \sigma t}\left|\sum_{j} a_{j}^{n} \varphi_{h}^{j}\right|_{H^{-1 / 2}(\Gamma)}^{2} \mathrm{~d} t
$$

en posant

$$
\left\|A^{n}\right\|=\left(\left|\sum_{j} a_{j}^{n} \psi_{h}^{j}\right|_{H^{-1 / 2}(\Omega)}^{2}\right)^{1 / 2}
$$

la norme de $A^{n}=\left(a_{j}^{n}\right)$ dans $\mathbf{R}^{N_{h}}\left(N_{h}=\right.$ dimension de $\left.\tilde{V}_{h}\right)$, on a donc

$$
\Delta t^{3} \frac{1-\mathrm{e}^{-2 \sigma \Delta t}}{2 \sigma} \sum_{n \geqslant 0} \mathrm{e}^{-2 \sigma t_{n}}\left\|A_{n}\right\|^{2} \leqslant \text { constante }
$$

ce qui donne aussi

$$
\text { (5.15) } \sum_{n \geqslant 0} \mathrm{e}^{-2 \sigma t} n\left\|\Delta t^{2} A^{n}\right\|^{2} \leqslant \text { constante. }
$$

Or, la relation (4.6) montre que le schéma proposé conduit en réalité à calculer les inconnues $A^{\prime n}=\Delta t^{3} A^{n}$ plutôt que $A^{n}$. La relation (5.15) implique clairement que si $\Delta t \rightarrow 0$ et $n \rightarrow+\infty$ de telle sorte que $0 \leqslant \Delta t n \leqslant T<\infty$, les $\left(A^{\prime n}\right)$ restent de l'ordre de $\Delta t$.

Si l'on mesurait par contre la norme $H^{0}(\Gamma)$ de $A^{n}$, tenant compte de (5.11), on aura des quantités calculées de l'ordre de $\frac{\Delta t}{\sqrt{h}}$. 
b) La constante $C$ explicitée dans (3.5) dans sa dépendance de $\sigma$ montre que la constante de (5.14) tend vers $\infty$ quand $\sigma \rightarrow 0$. On ne peut donc espérer faire $\sigma=0$. Ceci est valable pour toutes les estimations dans la suite.

\subsection{Estimation d'erreurs}

Théorème 5. Si la solution $\varphi$ du potentiel retardé vérifie la condition de régularité:

(5.16) $\varphi \in \boldsymbol{H}_{\sigma}^{2}\left(\mathbf{R}_{+}, H^{m_{1}+1}(\Gamma)\right) \cap \boldsymbol{H}_{\sigma}^{m_{2}+1}\left(\mathbf{R}_{+}, L^{2}(\Gamma)\right)$

on a l'estimation suivante pour la solution approchée:

$$
\begin{aligned}
\left|\varphi-\varphi_{h, \Delta t}\right|_{\sigma,-1 / 2,-1 / 2} \leqslant & C\left[\left|g_{h, \Delta t}^{\prime}-g^{\prime}\right|_{\sigma, 1 / 2,1 / 2}\right. \\
& \left.+h^{m_{1}+\frac{1}{2}}|\varphi|_{\sigma, 2, m_{1}+1}+\frac{\Delta t^{m_{2}}}{\sqrt{h}}|\varphi|_{\sigma, m_{2}+1,0}\right] .
\end{aligned}
$$

Démonstration. On part de la coercivité (3.5) appliquée à

$$
\begin{aligned}
& \varphi_{h, \Delta t}-\psi_{h, \Delta t} \in H_{\sigma}^{1}\left(\mathrm{R}_{+}, H^{-1 / 2}(\Gamma)\right) \text { où } \psi_{h, \Delta t} \in H_{\sigma}^{m_{2}}\left(\Delta t ; V_{h}\right): \\
& C\left|\varphi_{h, \Delta t}-\psi_{h, \Delta t}\right|_{\sigma,-1 / 2,-1 / 2}^{2} \leqslant b\left(\varphi_{h, \Delta t}-\psi_{h, \Delta t}, \varphi_{h, \Delta t}^{\prime}-\varphi^{\prime}\right) \\
& +b\left(\varphi_{h, \Delta t}-\psi_{h, \Delta t}, \varphi^{\prime}-\psi_{h, \Delta t}^{\prime}\right) \text {. }
\end{aligned}
$$

Tenant compte de (5.12) et (3.6), le ler terme est:

$$
b\left(\varphi_{h, \Delta t}-\psi_{h, \Delta t}, \varphi_{h, \Delta t}^{\prime}-\varphi\right)=\int_{0}^{\infty} \mathrm{e}^{-2 \sigma t} \int_{\Gamma}\left(\varphi_{h, \Delta t}-\psi_{h, \Delta t}\right)\left(g_{h, \Delta t}^{\prime}-g^{\prime}\right) \mathrm{d} \sigma_{x} \mathrm{~d} t
$$

qui est donc majorable, en passant par la formule de Plancheval, par

$$
\left|\varphi_{h, \Delta t}-\psi_{h, \Delta t}\right|_{\sigma,-1 / 2,-1 / 2}\left|g_{h, \Delta t}^{\prime}-g^{\prime}\right|_{\sigma, 1 / 2,1 / 2} \text {. }
$$

Tandis que, grâce à la proposition 4 , on peut majorer le $2^{d}$ terme au $2^{d}$ membre de (5.18) par:

$$
\begin{aligned}
& \mid b\left(\varphi_{h, \Delta t}-\psi_{h, \Delta t}, \varphi^{\prime}-\psi_{h, \Delta t}^{\prime}\right) \\
& \leqslant C\left|\varphi_{h, \Delta t}-\psi_{h, \Delta t}\right|_{\sigma,-1 / 2,0}\left|\varphi^{\prime}-\psi_{h, \Delta t}^{\prime}\right|_{\sigma, 1 / 2,0}
\end{aligned}
$$

ou en tenant compte de (5.11)

$$
\begin{aligned}
& \left|b\left(\varphi_{h, \Delta t}-\psi_{h, \Delta t}, \varphi^{\prime}-\psi_{h, \Delta t}^{\prime}\right)\right| \\
& \leqslant \frac{C}{\sqrt{h}}\left|\varphi_{h, \Delta t}-\psi_{h, \Delta t}\right|_{\sigma,-1 / 2,-1 / 2}\left|\varphi^{\prime}-\psi_{h, \Delta t}^{\prime}\right|_{\sigma, 1 / 2,0} .
\end{aligned}
$$

Finalement donc, on obtient:

$$
\begin{aligned}
\left|\varphi_{h, \Delta t}-\psi_{h, \Delta t}\right|_{\sigma,-1 / 2,-1 / 2} \leqslant & C\left[\left|g^{\prime}-g_{h, \Delta t}^{\prime}\right|_{\sigma, 1 / 2,1 / 2}\right. \\
& \left.+\frac{1}{\sqrt{h}}\left|\varphi^{\prime}-\psi_{h, \Delta t}^{\prime}\right|_{\sigma, 1 / 2,0}\right]
\end{aligned}
$$


ce qui donne par l'inégalité triangulaire

$$
\left\{\begin{array}{l}
\left|\varphi-\varphi_{h, \Delta t}\right|_{\sigma,-1 / 2,-1 / 2} \leqslant C\left[\left|g^{\prime}-g_{h, \Delta t}^{\prime}\right|_{\sigma, 1 / 2,1 / 2}\right. \\
+\inf _{\psi_{h, \Delta \in H_{\sigma}^{m}\left(\Delta t ; \bar{V}_{h}\right)}}\left\{\left|\varphi-\psi_{h, \Delta t}\right|_{\sigma,-1 / 2,-1 / 2}\right. \\
\left.\left.+\frac{1}{\sqrt{h}}\left|\varphi^{\prime}-\psi_{h, \Delta t}^{\prime}\right|_{\sigma, 1 / 2,0}\right\}\right] .
\end{array}\right.
$$

Il reste à majorer le inf. Grâce à (5.16), $\varphi \in C^{m_{2}}\left(\mathbf{R}_{+}, L^{2}(\Gamma)\right)$ et on peut définir:

$$
\psi_{h}(t, \cdot)=q_{h} \varphi(t, \cdot) \in C^{m_{2}}\left(\mathbf{R}_{+}, \tilde{V}_{h}\right) .
$$

Pour le lemme 4

$$
\left|\varphi(t, \cdot)-\psi_{h}(t, \cdot)\right|_{H^{-1 / 2}(I)} \leqslant C h^{m_{1}+\frac{3}{2}}|\varphi(t, \cdot)|_{H^{m_{1}+1}(I)} \quad \forall t \geqslant 0
$$

d'où

$$
\left|\varphi-\psi_{h}\right|_{\sigma, 0,-1 / 2} \leqslant C h^{m_{1}+\frac{3}{2}}|\varphi|_{\sigma, 0, m_{1}+1}
$$

comme on a aussi

$$
\int_{0}^{t} \psi_{h}(s) \mathrm{d} s=q_{h}\left(\int_{0}^{t} \varphi(s) \mathrm{d} s\right)
$$

la même inégalité est obtenue pour la primitive, d'où par interpolation:

$$
\left|\varphi-\psi_{h}\right|_{\sigma,-1 / 2,-1 / 2} \leqslant C h^{m_{1}+\frac{3}{2}}|\varphi|_{\sigma, 0, m_{1}+1} \text {. }
$$

On pose ensuite $\psi_{h, \Delta t}=r_{\Delta t} \psi_{h}$ d'après le lemme 3 (avec $k=m_{2}$ ), ce qui donne:

$$
\left|\psi_{h}-\psi_{h, \Delta t}\right|_{\sigma,-1 / 2,-1 / 2} \leqslant C \Delta t^{m_{2}+\frac{3}{2}}\left|\psi_{h}\right|_{\sigma, m_{2}+1,-1 / 2} \text {. }
$$

D'autre part, la définition même du projecteur $q_{h}$ donne:

$$
\left|\psi_{h}(t, \cdot)\right|_{H^{-1 / 2}(\Omega)} \leqslant\left|\psi_{h}(t, \cdot)\right|_{L^{2}(\Omega)} \leqslant|\varphi(t, \cdot)|_{L^{2}(I)}
$$

ainsi que des inégalités analogues pour les dérivées en $t$, d'où

$$
\left|\psi_{h}-\psi_{h, \Delta t}\right|_{\sigma,-1 / 2,-1 / 2} \leqslant C \Delta t^{m_{2}+\frac{3}{2}}|\varphi|_{\sigma, m_{2}+1,-1 / 2} \text {. }
$$

De même, on obtient:

$$
\begin{aligned}
& \left|\varphi^{\prime}-\psi_{h}^{\prime}\right|_{\sigma, \frac{1}{2}, 0} \leqslant C h^{m_{1}+1}\left|\varphi^{\prime}\right|_{\sigma, 1, m_{1}+1} \\
& \left|\psi_{h}^{\prime}-\psi_{h, \Delta t}^{\prime}\right|_{\sigma, 1 / 2,0} \leqslant C \Delta t^{m_{2}-\frac{1}{2}}|\varphi|_{\sigma, m_{2}+1,0}
\end{aligned}
$$

combinant le tout, on obtient: 


$$
\text { (5.20) }\left\{\begin{array}{l}
\inf _{\psi_{h, \Delta r} \in H_{\sigma}^{m_{2}\left(\Delta t ; \bar{V}_{h}\right)}}\left\{\left|\varphi-\psi_{h, \Delta t}\right|_{\sigma,-1 / 2,-1 / 2}+\frac{1}{\sqrt{h}}\left|\varphi^{\prime}-\psi_{h, \Delta t}^{\prime}\right|_{\sigma, 1 / 2,0}\right\} \\
\leqslant C\left[h^{m_{1}+\frac{1}{2}}\left(|\varphi|_{\sigma, 2, m_{1}+1}+h|\varphi|_{\sigma, 0, m_{1}+1}\right)\right. \\
\left.+\frac{\Delta t^{m_{2}-\frac{1}{2}}}{h^{1 / 2}}\left(|\varphi|_{\sigma, m_{2}+1,0}+h^{1 / 2} \Delta t^{2}|\varphi|_{\sigma, m_{2}+1,-1 / 2}\right)\right]
\end{array}\right.
$$

comme $|\varphi|_{\sigma, 0, m_{1}+1} \leqslant C|\varphi|_{\sigma, 2, m_{1}+1}$ et $|\varphi|_{\sigma, m_{2}+1,-1 / 2} \leqslant|\varphi|_{\sigma, m_{2}+1,0}$, il est clair que l'on obtient (5.17) en reportant (5.20) dे (5.19).

Remarque. On tire aisément de (2.13) une condition suffisante sur $g$ pour que $\varphi \in \boldsymbol{H}_{\sigma}^{m_{2}+1}\left(\mathbf{R}_{+}, L^{2}(I)\right)$. Pour la condition $\varphi \in H_{\sigma}^{2}\left(\mathbf{R}_{+}, H^{m_{1}+1}(I)\right)$, il faudra étudier de plus près l'opérateur $S_{\omega}$ qui est pour tout $\omega$, pseudo-différentiel d'ordre -1 sur $\Gamma$, afin de majorer en fonction de $\omega$ la norme $\left\|S_{\omega}^{-1}\right\|_{L\left(H^{m_{1}}+2(I), H^{m_{1}+1}(I)\right)}$.

Par des méthodes différentes, on obtient d'autres résultats de régularité voir Miyatake [16], Sakamoto [17]. Disons simplement que si $g$ est très régulière, (5.16) sera vérifiée.

Enfin, l'estimation (5.17) suggère que le "bon choix» des $m_{1}, m_{2}$ quand $\Delta t$ sont de même ordre, sera $m_{2}=m_{1}+1$.

En ce qui concerne l'erreur en norme $H_{\sigma}^{0}\left(\mathbf{R}_{+}, L^{2}(\Gamma)\right.$ ) (qui se calcule évidemment plus simplement que la norme $H_{\sigma}^{-1 / 2}\left(\mathrm{R}_{+}, H^{-1 / 2}(\Gamma)\right)$, on a le

Théorème 6. Si la solution $\varphi$ vérifie la condition de régularité

$$
\varphi \in H_{\sigma}^{1}\left(\mathbf{R}_{+}, \dot{H}^{m_{1}+1}(\Gamma)\right) \cap \boldsymbol{H}_{\sigma}^{m_{2}+1}\left(\mathbf{R}_{+}, L^{2}(\Gamma)\right)
$$

on $a:$

$$
\begin{aligned}
\left|\varphi-\varphi_{h, \Delta t}\right|_{\sigma, 0,0} \leqslant & C\left[\frac{1}{\sqrt{h} \cdot \sqrt{\Delta t}}\left|g_{h, \Delta t}^{\prime}-g^{\prime}\right|_{\sigma, 1 / 2,1 / 2}\right. \\
& \left.+\frac{h^{m_{1}+\frac{1}{2}}}{\sqrt{\Delta t}}|\varphi|_{\sigma, 1, m_{1}+1}+\frac{\Delta t^{m_{2}-\frac{1}{2}}}{\sqrt{h}}|\varphi|_{\sigma, m_{2}+1,0}\right] .
\end{aligned}
$$

Démonstration absolument analogue à celle du théorème 5 .

Passons maintenant à la solution $u$ de l'onde diffusée.

Posons pour $t>0, x \notin \Gamma$

$$
u_{h, \Delta t}(t, x)=\frac{1}{4 \pi} \int_{\Gamma} \frac{\varphi_{h, \Delta t}(t-|x-y|, y)}{|x-y|} \mathrm{d} \sigma_{y} .
$$

Nous pouvons essayer d'obtenir une majoration de

$$
\int_{0}^{\infty} \mathrm{e}^{-2 \sigma t}\left|u(t, x)-u_{h, \Delta t}(t, x)\right|^{2} \mathrm{~d} t
$$


pour $x$ fixé, dist $(x, I) \geqslant \delta>0$ en reprenant la majoration de Nédelec [11]. Mais ici, une majoration globale est possible avec l'énergie

$$
E(u)(t)=\int_{\Omega_{+}} \int_{\Omega_{-}}\left(|\nabla u(t, x)|^{2}+\left|\frac{\partial u}{\partial t}(t, x)\right|^{2}\right) d x .
$$

Théorème 7. Nous avons dans les conditions du théorème 5:

$$
\begin{aligned}
& \int_{0}^{\infty} \mathrm{e}^{-2 \sigma t} E\left(u-u_{h, \Delta t}\right)(t) \mathrm{d} t \\
& \leqslant C\left(\left|g_{h, \Delta t}^{\prime}-g^{\prime}\right|_{\sigma, 1 / 2,1 / 2}^{2}+\left|g_{h, \Delta t}^{\prime}-g^{\prime}\right|_{\sigma, 1 / 2,1 / 2}\right. \\
& \left.\quad\left(h^{m_{1}+\frac{1}{2}}|\varphi|_{\sigma, 2, m_{1}+1}+\frac{\Delta t^{m_{2}}}{\sqrt{h}}|\varphi|_{\sigma, m_{2}+1,0}\right)\right) .
\end{aligned}
$$

Il suffit en effet d'appliquer la formule de Plancherel d̀ l'égalite de Green (2.13) où $S \varphi$ est remplacé par $g$.

\$6 Analyse du schema avec approximation de surface

Au lieu donc du problème (5.11), nous aurons à déterminer

$$
f_{h, \Delta t}=\varphi_{h, \Delta t}^{\prime} \in H_{\sigma}^{m_{2}-1}\left(\Delta t ; V_{h}\right)
$$

solution de

$$
\left\{\begin{array}{l}
b_{h}\left(\psi_{h, \Delta t}, f_{h, \Delta t}\right)=\int_{0}^{\infty} \mathrm{e}^{-2 \sigma t} \int_{\Gamma_{h}} \psi_{h, \Delta t}(t, x) g_{h, \Delta t}^{\prime}(t, x) \mathrm{d} \sigma_{x} \mathrm{~d} t \\
\forall \psi_{h, \Delta t} \in H_{\sigma}^{m_{2}}\left(\Delta t ; V_{h}\right)
\end{array}\right.
$$

où la forme $b_{h}$ est:

$$
\begin{aligned}
& b_{h}\left(\psi_{h, \Delta t}, f_{h, \Delta t}\right) \\
& =\int_{0}^{\infty} \mathrm{e}^{-2 \sigma t} \iint_{\Gamma_{h} \times \Gamma_{h}} \frac{\psi_{h, \Delta t}(t, x) f_{h, \Delta t}(t-|x-y|, y)}{4 \pi|x-y|} \mathrm{d} \sigma_{x} \mathrm{~d} \sigma_{y} \mathrm{~d} t .
\end{aligned}
$$

Avec les notations des $\S 4,5$, on peut ramener les intégrales sur $\Gamma_{h}$ à des intégrales sur $\Gamma$ par l'application $P^{-1}$. Les deux membres de (6.2) deviennent alors

$$
\begin{aligned}
& \bar{b}_{h}\left(\tilde{\psi}_{h, \Delta t}, \tilde{f}_{h, \Delta t}\right) \\
& =\int_{0}^{\infty} \mathrm{e}^{-2 \sigma t} \iint_{\Gamma \times \Gamma} \frac{\tilde{\psi}_{h, \Delta t}(t, \tilde{x}) \tilde{f}_{h, \Delta t}\left(t-\left|P^{-1} \tilde{x}-P^{-1} \tilde{y}\right|, \tilde{y}\right)}{4 \pi\left|P^{-1} \tilde{x}-P^{-1} \tilde{y}\right|} \\
& \quad \cdot J(\tilde{x}) J(\tilde{y}) \mathrm{d} \sigma_{\bar{x}} \mathrm{~d} \sigma_{\tilde{y}} \mathrm{~d} t
\end{aligned}
$$

où $J$ est le déterminant jacobien de $P^{-1}$. 
Les fonctions $\tilde{\psi}_{h, \Delta t}$ et $\tilde{f}_{h, \Delta t}$ appartiennent maintenant respectivement $\boldsymbol{H}_{\sigma}^{m_{2}}\left(\Delta t ; \tilde{V}_{h}\right)$ et $\boldsymbol{H}_{\sigma}^{m_{2}-1}\left(\Delta t ; \bar{V}_{h}\right)$.

Nous avons donc modifié le problème (5.11) en changeant la forme bilinéaire $b$ en la forme $\tilde{b}_{h}$, ainsi qu'un changement analogue du second membre.

Nous commençons par estimer la différence $\bar{b}_{h}-b$.

Par transformation de Laplace, nous devons regarder la différence entre

$$
a^{\omega}(\psi, f)=\iint_{\Gamma \times \Gamma} \frac{e^{\mathrm{i} \omega|x-y|} f(y) \bar{\psi}(x)}{|x-y|} \mathrm{d} \sigma_{x} \mathrm{~d} \sigma_{y}
$$

et

$$
a_{h}^{\omega}(\psi, f)=\iint_{\Gamma \times \Gamma} \frac{e^{\mathrm{i} \omega\left|P^{-1} x-P^{-1} y\right|} f(y) \bar{\psi}(x)}{\left|P^{-1} x-P^{-1} y\right|} J(x) J(y) \mathrm{d} \sigma_{x} \mathrm{~d} \sigma_{y}
$$

avec $\psi, f \in \tilde{V}_{h}$ complexe.

En suivant les calculs de Nédelec [11], on décompose la différence $a^{\omega}-a_{h}^{\omega}$ en trois termes $R_{1}+R_{2}+R_{3}$, avec:

$$
\begin{aligned}
& R_{1}=\iint_{\Gamma \times \Gamma} \frac{\mathrm{e}^{\mathrm{i} \omega|x-y|}}{|x-y|} \bar{\psi}(x) f(y)(1-J(x) J(y)) \mathrm{d} \sigma_{x} \mathrm{~d} \sigma_{y}
\end{aligned}
$$

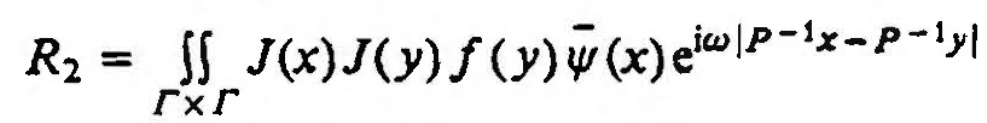

$$
\begin{aligned}
& \cdot\left(\frac{1}{|x-y|}-\frac{1}{\left|P^{-1} x-P^{-1} y\right|}\right) \mathrm{d} \sigma_{x} \mathrm{~d} \sigma_{y} \\
& R_{3}=\iint_{\Gamma \times \Gamma} J(x) J(y) f(y) \vec{\psi}(x) \frac{1}{|x-y|}\left(\mathrm{e}^{\mathrm{i} \omega|x-y|}-\mathrm{e}^{\mathrm{i} \omega \mid P-1 x-P-1} y \mid\right) \mathrm{d} \sigma_{x} \mathrm{~d} \sigma_{y} .
\end{aligned}
$$

Les calculs de [11] s'appliquent sans changement à $R_{1}, R_{2}$ oul l'on pouvait majorer l'exponentiel par 1 si Im $\omega \geqslant 0$, et à $R_{3}$ après la majoration obtenue par le theorème des accroissements finis:

$$
\left|e^{\mathrm{i} \omega|x-y|}-\mathrm{e}^{\mathrm{i} \omega\left|P^{-1} x-P^{-1} y\right|}\right| \leqslant|| x-y|-| P^{-1} x-P^{-1} y|| \cdot|\omega| .
$$

On obtient enfin:

$$
\left\{\begin{array}{l}
\left|a^{\omega}(\psi, f)-a_{h}^{\omega}(\psi, f)\right| \leqslant C|\omega| h^{m_{0}+\frac{1}{2}}|\psi|_{-\frac{1}{2}}|f|_{0} \\
\leqslant C^{\prime}|\omega| h^{m_{0}}|\psi|_{-\frac{1}{2}}|f|_{-\frac{1}{2}} \\
\text { pour tout } \psi, f \in \bar{V}_{h} \text { complexe, Im } \omega \geqslant \sigma_{0}>0 \text { et } 0<h \leqslant h_{0} .
\end{array}\right.
$$

La constante $C$ comme toujours ne dépend que de $\sigma_{0}$ (et de $\Gamma$ ). L'entier $m_{0}$ est celui de l'élément fini servant à construire la surface $\Gamma_{h}$. On en déduit le

Lemme 5. Si $h^{m_{0}} / \Delta t^{3}$ est suffisamment petit, nous avons pour $\tilde{b}_{h}$ l'inégalité de coercivité suivante, analogue d celle de b:

$$
\begin{aligned}
& \tilde{b}_{h}\left(\varphi_{h, \Delta t}, \varphi_{h, \Delta t}^{\prime}\right) \geqslant C\left|\varphi_{h, \Delta t}\right|_{\sigma,-1 / 2,-1 / 2}^{2} \\
& \text { pour tout } \varphi_{h, \Delta t} \in H_{\sigma}^{k}\left(\Delta t ; \tilde{V}_{h}\right), k \geqslant 1 .
\end{aligned}
$$


En effet on obtient de (6.3) par la formule de Plancherel:

$$
\left|b\left(\psi_{h, \Delta t}, f_{h, \Delta t}\right)-\tilde{b}_{h}\left(\psi_{h, \Delta t}, f_{h, \Delta t}\right)\right| \leqslant C_{1} h^{m_{0}}\left|\psi_{h, \Delta t}\right|_{\sigma, 1,-\frac{1}{2}}\left|f_{h, \Delta t}\right|_{\sigma, 0,-1 / 2}
$$

soit avec $\psi_{h, \Delta t}=\varphi_{h, \Delta t}$ et $f_{h, \Delta t}=\varphi_{h, \Delta t}^{\prime}$

$$
\left|b\left(\varphi_{h, \Delta t}, \varphi_{h, \Delta t}^{\prime}\right)-\tilde{b}_{h}\left(\varphi_{h, \Delta t}, \varphi_{h, \Delta t}^{\prime}\right)\right| \leqslant C_{1} h^{m_{0}}\left|\varphi_{h, \Delta t}\right|_{\sigma, 1,-1 / 2}^{2}
$$

(5.3) permet ensuite de majorer le dernier terme par $C_{2} h^{m_{0} \Delta t^{-3}}\left|\varphi_{h, \Delta t}\right|_{\sigma,-1 / 2,-1 / 2}^{2}$ tandis que $b\left(\varphi_{h, \Delta t}, \varphi_{h, \Delta t}^{\prime}\right) \geqslant C_{3}\left|\varphi_{h, \Delta t}\right|_{\sigma,-1 / 2,-1 / 2}^{2}$. On voit qu'il suffit que $h^{m_{0}} \Delta t^{-3} \leqslant C_{3} / 2 C_{2}$ pour avoir (6.4) avec $C=C_{3} / 2$.

L'estimation d'erreurs du schéma (1) peut alors se faire de la même façon qu'au $\S 5$, avec un terme supplémentaire do au changement de forme bilinéaire.

Théorème 8. Sous les conditions du lemme 5 , et si la solution $\varphi$ vérifie la régularité (5.16), on a les estimations suivantes pour la solution $\varphi_{h, \Delta t}$ du schéma (6.1):

$$
\begin{aligned}
& \left\{\begin{aligned}
\left|\varphi-\tilde{\varphi}_{h, \Delta t}\right|_{\sigma,-1 / 2,-1 / 2} \leqslant & C\left[\left|\tilde{g}_{h, \Delta t}^{\prime}-g^{\prime}\right|_{\sigma, 1 / 2,1 / 2}\right. \\
& +h^{m_{1}+\frac{1}{2}}|\varphi|_{\sigma, 2, m_{1}+1}+\frac{\Delta t^{m_{2}}}{\sqrt{h}}|\varphi|_{\sigma, m_{2}+1,0} \\
& \left.+\frac{h^{m_{0}+\frac{1}{2}}}{\Delta t^{3 / 2}}\left|\varphi^{\prime}\right|_{\sigma, 0,0}\right] \\
& +\frac{h^{m_{1}+\frac{1}{2}}}{\sqrt{\Delta t}}|\varphi|_{\sigma, 1, m_{1}+1}+\frac{\Delta t^{m_{2}-\frac{1}{2}}}{\sqrt{h}}|\varphi|_{\sigma, m_{2}+1,0} \\
& \left.+\frac{h^{m_{0}}}{\Delta t^{2}}|\varphi|_{\sigma, 0,0}\right] .
\end{aligned}\right.
\end{aligned}
$$

Démonstration. On reprend exactement les mêmes relations que dans la démonstration des th. 5, 6 avec $\tilde{\varphi}_{h, \Delta t}, \tilde{\psi}_{h, \Delta t}$ à la place de $\varphi_{h, \Delta t}, \psi_{h, \Delta t}$ etc. . . Le terme supplémentaire qui s'introduit à cause du changement de la forme bilinéaire se majore grâce à (6.3)

$$
\begin{aligned}
& \frac{\left|b\left(\tilde{\varphi}_{h, \Delta t}-\tilde{\psi}_{h, \Delta t}, \tilde{\psi}_{h, \Delta t}^{\prime}\right)-\tilde{b}_{h}\left(\tilde{\varphi}_{h, \Delta t}-\tilde{\psi}_{h, \Delta t}, \tilde{\psi}_{h, \Delta t}^{\prime}\right)\right|}{\left|\tilde{\varphi}_{h, \Delta t}-\tilde{\psi}_{h, \Delta t}\right|_{\sigma,-\frac{1}{2},-\frac{1}{2}}} \\
& \leqslant C h^{m_{0}+\frac{1}{2}}\left|\tilde{\psi}_{h, \Delta t}^{\prime}\right|_{\sigma, \frac{3}{2}, 0} \leqslant C \frac{h^{m_{0}+\frac{1}{2}}}{\Delta t^{\frac{3}{2}}}\left|\tilde{\psi}_{h, \Delta t}^{\prime}\right|_{\sigma, 0,0}
\end{aligned}
$$

si $m_{2} \geqslant 2$, d'après le corollaire du lemme 2 . 
Le choix de $\vec{\psi}_{h, \Delta t}$ dans la démonstration du théorème 5 permet ensuite de majorer $\left|\tilde{\psi}_{h, \Delta t}^{\prime}\right|_{\sigma, 0,0} \operatorname{par}\left|\varphi^{\prime}\right|_{\sigma, 0,0}$. D'où (6.5). De même pour (6.6).

Ce résultat n'est évidemment pas otpimal. En particulier, pour une surface d'ordre 2 , le choix de $m_{0}=2$ correspond à $\Gamma_{h}=\Gamma$, c'est-à-dire à la surface exacte! La condition draconienne du lemme 5 n'est donc pas à vérifier. On peut donc supposer que l'estimation du terme d'erreurs dûes au changement de géométrie (terme correspondant à la différence des formes bilinéaires $b$ et $b_{h}$ ) est sans doute exagérée et dûe essentiellement à la méthode choisie. Néanmoins, nous ne sommes pas parvenus à la surmonter. D'un autre côté, il semble quand même normal que des phénomènes de réflexions puissent rendre déterminante cette erreur de géométrie.

En se restreignant aux obstacles convexes, ou en considérant les termes de courbure dans l'approximation de surfaces, peut-être arrivera-t-on à améliorer ce résultat?

En tout cas, (6.5) reste quand même un résultat de convergence du schéma quand $h$ et $\Delta t$ tendent tous deux vers 0 en gardant constant un certain rapport entre eux.

\section{Conclusion}

Nous avons ainsi présenté et analysé une nouvelle classe de schémas de calculs pour l'équation du potentiel retardé. Le caractère convolutif de ces schémas (cf. 4.5) exigera bien entendu beaucoup de mémoires d'ordinateurs dans une mise en oeuvre (restant à faire). Mais c'est évidemment inévitable, car intrinsèque. De plus notre schéma est quasiment explicite: une seule matrice est à inverser, et le sera donc une fois pour toutes. Les autres matrices intervenant dans (4.5) sont aussi calculées une seule fois, et réutilisées aux pas de temps suivants. En outre, avec la formulation variationnelle (3.6), c'est la première fois à notre connaissance que des résultats de stabilité et de convergence sont obtenus dans ce problème.

\section{Bibliographie}

\section{Applications du potentiel retardé}

[1] Bennett, C. L.; Mieras, H.: Time domain integral equation solution for acoustic scattering from fluid targets. JASA 59, n 5 (1981) $1261-1265$

[2] Bennett, C. L.; Ross, G. F.: Time domain electromagnetics and its applications. Proc. I.E.E.E. $66, \mathrm{n}^{\circ} 3$ (1978) $299-318$

[3] Cole, D. M.; Kosloff, D. D.; Minster, J. B.: A numerical boundary integral equation method for elastodynamics I. Bull. Seism. Soc. Am. 68, $n^{\circ} 5$ (1978) $1331-1357$

[4] Herma, G. C.: Scattering of transient acoustic waves by an inhomogeneous obstacle. JASA 69, $n^{\circ} 4$ (1981) $909-915$

[5] Herman, G. C.: Scattering of transient elastic waves by an inhomogeneous obstacle: contrast in volume density of mass. JASA $71, n^{\circ} 1$ (1982) $264-272$

[6] Mitzner, K. M.: Numerical solution of transient scattering from a hard surface of arbitrary shape - retarded potential technique. JASA 42, nº 2 (1967) 391 - 397 
[7] Parot, J. M.; Pages, J. M.; Verpeau, P.: Calcul des surpressions exercées par une onde acoustique aérienne sur des structures réfléchissantes. Note technique DEMT/SMTS/LAMS $83-35$

[8] Shaw, R. P.: Boundary integral equation methods applied to wave problems. In: Developpements in boundary element Methods, ed. by Banerjee, $P$. and Butterfield, $R$. London: Elsevier 1979

[9] Shaw, R. P.; English, J. A.: Transient acoustic scattering by a free (pressure release) sphere. J. Sound and Vibration 20, $\mathrm{n}^{\circ} 3$ (1972) $321-331$

\section{Approximation des équations intégrales}

[10] Giroire, J.: Integral equation methods for extension problems for the Helmholtz Equation. Rapport interne $n^{\circ} 40$ (1978), Centre de Mathématiques Appliquées, Ecole Polytechnique, Palaiseau

[11] Nedelec, J. C.: Approximation des équations intégrales en mécanique et en physique. Cours de l'Ecole d'Eté d'Analyse Numérique EDF-CEA-INRIA (1977)

[12] Nedelec, J. C.; Planchard, J.: Une méthode variationnelle d'éléments finis pour la résolution numérique d'un problème extérieur dans $R^{3}$. R.A.I.R.O., R3, 7 (1973) $105-129$

\section{Equations des ondes}

[13] Charazain, J.; Piriou, A.: Introduction à la theorie des equations aux dérivées partielles. Paris: Gauthiers Villards 1981

[14] Lebeau, G.; Schatzman, M.: A wave problem in a half-space with a one-sided constraint at the boundary. Rapport interne $n^{\circ} 84$ (1982), Centre de Mathématiques Appliquées, Ecole Polytechnique, Palaiseau

[15] Lions, J. L.; Magenes, E.: Problèmes aux limites non homogènes et applications, vol. 1, 2. Paris: Dunod 1968

[16] Miyatake, Sadas.: Mixed problems for hyperbolic equations of second order. J. Math. Kyote University 13 (1973) $435-487$

[17] Sakam ot o, Reiko.: Mixed problems for hyperbolic equations I, II. J. Math. Kyoto University 10 (1970) $349-373$ et $375-401$

[18] Treves, P.: Basic linear partial differential equations. New York: Academic Press 1975
A. Bamberger
T. Ha Duong
Ecole Polytechnique
Centre de Mathématiques Appliquées
F-91128 Palaiseau Cedex 